\title{
DISTANCE TO THE CONVEX HULL OF AN ORBIT UNDER THE ACTION OF A COMPACT LIE GROUP
}

\author{
RANDALL R. HOLMES and TIN-YAU TAM
}

(Received 12 June 1998; revised 29 January 1999)

Communicated by A. H. Dooley

\begin{abstract}
For a real vector space $V$ acted on by a group $K$ and fixed $x$ and $y$ in $V$, we consider the problem of finding the minimum (respectively, maximum) distance, relative to a $K$-invariant convex function on $V$, between $x$ and elements of the convex hull of the $K$-orbit of $y$. We solve this problem in the case where $V$ is a Euclidean space and $K$ is a finite reflection group acting on $V$. Then we use this result to obtain an analogous result in the case where $K$ is a maximal compact subgroup of a reductive group $G$ with adjoint action on the vector component $p$ of a Cartan decomposition of Lie $G$. Our results generalize results of $\mathrm{Li}$ and $\mathrm{Tsing}$ and of Cheng concerning distances to the convex hulls of matrix orbits.
\end{abstract}

1991 Mathematics subject classification (Amer. Math. Soc.): primary 17B20, 22 E46.

Keywords and phrases: Distance, convex hull, orbit, reductive Lie group.

\section{Introduction}

In [LT2], Li and Tsing studied the distance to the convex hull of the orbit of a Hermitian matrix under the conjugacy action of the unitary group. We begin by describing their results. Let $H_{n}$ denote the space of $n \times n$ Hermitian matrices and let $U(n)$ denote the group of $n \times n$ unitary matrices. Fix $B \in H_{n}$. Denote by $O(B)$ the orbit of $B$ under the conjugacy action of $U(n)$, that is, $O(B)=\left\{U B U^{-1}: U \in U(n)\right\}$, and let $C(O(B))$ denote the convex hull of this orbit. Let $\lambda(B)=\left(\lambda_{1}(B), \ldots, \lambda_{n}(B)\right)$ be the eigenvalues of $B$ arranged in nonincreasing order. Li and Tsing showed that, given any unitary similarity invariant norm $\|\cdot\|: H_{n} \rightarrow \mathbb{R}$ (meaning norm that is constant on orbits) and any $A \in H_{n}$, one has

$$
\max \{\|A-X\|: X \in C(O(B))\}=\left\|\operatorname{diag}\left(\lambda_{1}(A)-\lambda_{n}(B), \ldots, \lambda_{n}(A)-\lambda_{1}(B)\right)\right\| .
$$

(c) 1999 Australian Mathematical Society 0263-6115/99 \$A2.00+0.00 
So this gives a formula in terms of eigenvalues for finding the maximum distance (relative to $\|\cdot\|$ ) between $A \in H_{n}$ and elements in the convex hull of the orbit of $B$. Li and Tsing also found a formula for the minimum such distance. It involves an algorithm of at most $n$ iterations starting with $\lambda(A)$ and $\lambda(B)$.

Following the lead of $\mathrm{Li}$ and Tsing, Cheng studied in $[\mathrm{C}]$ the problem of finding extreme distances to convex hulls of orbits in other matrix settings. Here is one such setting he considered.

Let $\mathbb{C}_{m \times n}$ be the space of $m \times n$-matrices over $\mathbb{C}$. Then there is an action of the group $U(m) \times U(n)$ on $\mathbb{C}_{m \times n}$ given by $(U, V) \cdot B=U B V^{*}$. Denote again by $O(B)$ the orbit of $B \in \mathbb{C}_{m \times n}$ under this action. Cheng found formulas similar to those of $\mathrm{Li}$ and Tsing for the extremes of $\{\|A-X\|: X \in C(O(B))\}$, where $A, B \in \mathbb{C}_{m \times n}$ and $\|\cdot\|$ is an invariant norm.

As another example, let $K_{n}(\mathbb{R})$ denote the space of $n \times n$ skew symmetric matrices over $\mathbb{R}$. Then the group $O_{n}(\mathbb{R})$ of real orthogonal matrices acts on $K_{n}(\mathbb{R})$ by the rule $O \cdot X=O X O^{t}$. Cheng found formulas for the extreme distances (relative to an invariant norm) to elements in the convex hull of the orbit of a given $B \in K_{n}(\mathbb{R})$, and these formulas again resemble those for the other cases we have discussed. Additional matrix settings were considered by Cheng and the findings were all similar.

Looking at the results of $\mathrm{Li}$ and Tsing and of Cheng, it is natural to ask whether there might be a unified approach for studying these problems. The purpose of this paper is to present such a unified approach.

We begin in Section 2 by considering a finite reflection group $W$ acting on a Euclidean space $E$. Given $x, y \in E$ and a $W$-invariant convex function $\varphi: E \rightarrow \mathbb{R}$, we compute the extremes of the set $\{\varphi(x-z): z \in C(W y)\}$. (See Theorem 2.13.)

In Section 3 we turn to the study of a reductive Lie group $G$, or more precisely, an element $(G, K, \theta, B)$ of the Harish-Chandra class. The compact Lie group $K$ acts naturally on the vector space $\mathfrak{p}$, where $\mathfrak{g}=\mathfrak{k}+\mathfrak{p}$ is the Cartan decomposition of $\mathfrak{g}=$ Lie $G$ corresponding to $\theta$. We use Kostant's convexity theorem as well as a classical result of Berezin and Gel'fand (both of which we extend to the case of a reductive Lie group) to show that, for a $K$-invariant convex function $\varphi: \mathfrak{p} \rightarrow \mathbb{R}$ and for $x, y \in \mathfrak{p}$, the set $\{\varphi(x-z): z \in C(K y)\}$ has the same extremes as the set $\{\varphi(\bar{x}-z): z \in C(W \bar{y})\}$, where $\bar{x}$ is the unique element in the intersection of the orbit $K x$ and a certain Euclidean space $\mathfrak{a} \subseteq \mathfrak{p}$ (and similarly for $\bar{y}$ ) and $W$ is a finite reflection group acting on $\mathfrak{a}$ ( $W$ is the Weyl group of the pair $(\mathfrak{g}, \mathfrak{a})$ ). The results of Section 2 then apply to give these extremes. (See Theorem 3.12.)

In Section 3 we also generalize to the reductive Lie group setting a theorem of $\mathrm{Li}$ and Tsing [LT1] concerning unitary similarity invariant norms on the set of $n \times n$ Hermitian matrices as well as a characterization given by von Neumann [vN] of unitarily invariant norms on $\mathbb{C}_{m \times n}$. (See Theorem 3.8, Example 4.1 and Example 4.3.)

Finally, we show in Section 4 that the results of $\mathrm{Li}$ and Tsing and the results of 
Cheng can all be recovered from our general results. In particular, we show that an algorithm we obtain for finding the minimum of the set $\{\varphi(x-z): z \in C(K y)\}$ generalizes these authors' algorithms.

\section{Results for finite reflection groups}

In this section, we obtain a means for computing the minimum and maximum distances to the convex hull of an orbit under the the action of a finite reflection group. The main result, Theorem 2.13, will be used in the next section to obtain an analog, Theorem 3.12, pertaining to the convex hull of an orbit under the action of a compact Lie group. We begin with some definitions and standard results from the theory of finite reflection groups. (For more details, see [BGr, H2].)

Let $E$ be a (real) finite dimensional Euclidean space with associated inner product $(\cdot, \cdot)$. Given a nonzero element $\alpha$ of $E$, denote by $s_{\alpha}: E \rightarrow E$ the reflection in the hyperplane orthogonal to $\alpha: s_{\alpha}(x)=x-\langle x, \alpha\rangle \alpha$, where $\langle x, \alpha\rangle:=2(x, \alpha) /(\alpha, \alpha)$.

Fix a root system $\Phi \subseteq E$. By definition, $\Phi$ is a finite set of nonzero vectors such that, for each $\alpha \in \Phi$,

(1) $\Phi \cap \mathbb{R} \alpha=\{\alpha,-\alpha\}$, and

(2) $s_{\alpha} \Phi=\Phi$.

Associated with $\Phi$ is the 'finite reflection group' $W$ generated by the reflections $s_{\alpha}$ $(\alpha \in \Phi)$.

Let $E_{1}$ denote the $\mathbb{R}$-span of $\Phi$ and let $E_{0}$ denote its orthogonal complement in $E$. Fix a simple system $\Delta=\left\{\alpha_{1}, \ldots, \alpha_{n}\right\} \subseteq \Phi$. Then $\Delta$ is a basis for $E_{1}$ such that $\Phi=\Phi^{+} \dot{U}\left(-\Phi^{+}\right)$, where $\Phi^{+}:=\left\{\alpha \in \Phi: \alpha=\sum_{i} a_{i} \alpha_{i}\right.$ with $\left.a_{i} \geq 0\right\}$. It is shown that $W$ is generated by $\left\{s_{\alpha_{i}}\right\}$.

Let $\lambda_{1}, \ldots, \lambda_{n} \in E$ be the vectors satisfying $\left\langle\lambda_{j}, \alpha_{i}\right\rangle=\delta_{i j}$ (Kronecker delta) (so $\left\{\lambda_{i}\right\}$ is just the basis of $E_{1}$ dual to the basis $\left\{2 \alpha_{i} /\left(\alpha_{i}, \alpha_{i}\right)\right\}$ relative to the inner product). The matrix $\left(\left\langle\alpha_{i}, \alpha_{j}\right\rangle\right)$ is called the Cartan matrix. It is the change of basis matrix from $\left\{\lambda_{j}\right\}$ to $\left\{\alpha_{i}\right\}: \alpha_{i}=\sum_{j}\left\langle\alpha_{i}, \alpha_{j}\right\rangle \lambda_{j}$. The off-diagonal entries of the Cartan matrix are nonpositive, that is, $\left\langle\alpha_{i}, \alpha_{j}\right\rangle \leq 0$ for $i \neq j$. Let $\left(d_{j i}\right)$ denote the inverse of the Cartan matrix.

Set $N=\{1, \ldots, n\}$ and let $I \subseteq N$. The set $\Phi_{I}=\Phi \cap \operatorname{span}\left\{\alpha_{i}: i \in I\right\}$ is a root system in $E$ with simple system $\Delta_{I}=\left\{\alpha_{i}: i \in I\right\}$. Denote the associated finite reflection group by $W_{I}$ and denote the inverse of the Cartan matrix $\left(\left\langle\alpha_{i}, \alpha_{j}\right\rangle\right)_{i, j \in I}$ by $\left(d_{j i}^{l}\right)$.

Let $\mathscr{L}$ be the collection of all subsets $L$ of $N$ for which there does not exist a nonempty subset $J \subsetneq L$ satisfying $\left(\alpha_{j}, \alpha_{k}\right)=0$ for all $j \in J, k \in L \backslash J$. So $L \in \mathscr{L}$ if and only if $\Phi_{L}$ is irreducible in the sense of [BGr, p. 56].

A proof of the following result is sketched in [H1, p. 72, Exercises 7 and 8]. 
LEMMA 2.1. (1) $d_{j i} \geq 0$ for all $i, j \in N$.

(2) If $L \in \mathscr{L}$, then $d_{j i}>0$ for all $i, j \in L$.

For $I \subseteq N$ set

$$
\begin{aligned}
& E^{-}(I)=\left\{x \in E_{1}:\left(x, \lambda_{i}\right)=0 \text { for all } i \notin I \text { and }\left(x, \lambda_{i}\right)<0 \text { for all } i \in I\right\}, \\
& E^{+}(I)=\left\{x \in E:\left(x, \alpha_{j}\right)=0 \text { for all } j \in I \text { and }\left(x, \alpha_{j}\right) \geq 0 \text { for all } j \notin I\right\} .
\end{aligned}
$$

LEMMA 2.2. Let $x \in E$ and $I \subseteq N$.

(1) $x \in E^{-}(I)$ if and only if $x=\sum_{i \in I} a_{i} \alpha_{i}$ with $a_{i}<0$.

(2) $x \in E^{+}(I)$ if and only if $x=x^{0}+\sum_{j \not I} b_{j} \lambda_{j}$ with $x^{0} \in E_{0}, b_{j} \geq 0$.

(3) $\left(E^{-}(I), E^{+}(I)\right)=0$.

ProOF. Assume $x \in E_{1}$. We will prove the lemma for this special case (with $x^{0}=0$ in (2)); the general case will then follow. Since $\left\{\alpha_{i}\right\}$ and $\left\{\lambda_{j}\right\}$ are both bases for $E_{1}$, we can write $x$ in the form $\sum_{i} a_{i} \alpha_{i}=x=\sum_{j} b_{j} \lambda_{j}\left(a_{i}, b_{j} \in \mathbb{R}\right)$. Applying $\left(\cdot, \lambda_{i}\right)$ yields $a_{i}=2\left(x, \lambda_{i}\right) /\left(\alpha_{i}, \alpha_{i}\right)$ while applying $\left\langle\cdot, \alpha_{j}\right\rangle$ yields $b_{j}=\left\langle x, \alpha_{j}\right\rangle$. This proves (1) and (2). Since $\left(\lambda_{j}, \alpha_{i}\right)=0$ for $i \neq j,(3)$ now follows from (1) and (2).

For $x \in E$ and $I \subseteq N$, set

$$
x(I)=\sum_{i \in I} c_{i}^{l}(x) \alpha_{i}
$$

where

$$
c_{i}^{I}(x)=\sum_{j \in l} d_{j i}^{I}\left\langle x, \alpha_{j}\right\rangle \quad(i \in I)
$$

It follows from Lemma 2.3 (1) below that $x(I)$ is the orthogonal projection of $x$ onto $E_{I}=\operatorname{span} \Phi_{I}$, and hence $c_{i}^{l}(x)$ is the $i$ th coordinate of this projection relative to the basis $\Delta_{I}$ of $E_{I}$.

LEMMA 2.3. Let $x \in E$ and $I \subseteq N$.

(1) $\left(x-x(I), \alpha_{i}\right)=0$ for all $i \in I$.

(2) $\left(x(I), \lambda_{i}\right)= \begin{cases}c_{i}^{l}(x)\left(\alpha_{i}, \alpha_{i}\right) / 2, & i \in I \\ 0, & i \notin I .\end{cases}$

PROOF. (1) Let $i \in I$. From the definitions of $x(I)$ and $d_{j k}^{l}$, we obtain

$$
\begin{aligned}
\left\langle x-x(I), \alpha_{i}\right\rangle & =\left\langle x, \alpha_{i}\right\rangle-\sum_{j, k \in I} d_{j k}^{I}\left\langle x, \alpha_{j}\right\rangle\left\langle\alpha_{k}, \alpha_{i}\right\rangle \\
& =\left\langle x, \alpha_{i}\right\rangle-\sum_{j \in I}\left\langle x, \alpha_{j}\right\rangle \delta_{i j}=0,
\end{aligned}
$$


whence $\left(x-x(I), \alpha_{i}\right)=0$.

(2) Let $i \in N$. Applying $\left(\cdot, \lambda_{i}\right)$ to the definition of $x(I)$, we get

$$
\left(x(I), \lambda_{i}\right)=\sum_{j, k \in I} d_{j k}^{I}\left\langle x, \alpha_{j}\right\rangle\left(\alpha_{k}, \lambda_{i}\right)
$$

Since $\left(\alpha_{k}, \lambda_{i}\right)=\delta_{i k}\left(\alpha_{k}, \alpha_{k}\right) / 2$, the claim follows.

Next, we describe an algorithm that will play a key role in the remainder of the paper.

AlgORITHM 2.4. Let $x \in E$ and set $x_{0}=x, I_{0}=\phi$. Suppose $x_{k-1}$ and $I_{k-1}(k \in \mathbb{N})$ have been defined. If $\left(x_{k-1}, \alpha_{l}\right) \geq 0$ for all $l$, then the algorithm ends. Otherwise, choose $I_{k} \subseteq N$ such that $I_{k} \supsetneq I_{k-1}$ and

(1) $c_{i}^{l_{k}}\left(x_{k-1}\right) \leq 0$ for all $i \in I_{k}$, and

(2) $c_{i}^{I_{k}}\left(x_{k-1}\right)<0$ for all $i \in I_{k} \backslash I_{k-1}$.

Then set $x_{k}=x_{k-1}-x_{k-1}\left(I_{k}\right)$.

We need to show that such an $I_{k}$ always exists. Assume $\left(x_{k-1}, \alpha_{l}\right)<0$ for some $l$ and set $I_{k}=I_{k-1} \cup\{l\}$. By Lemma $2.3(1),\left(x_{k-1}, \alpha_{i}\right)=0$ for all $i \in I_{k-1}$. In particular, $l \notin I_{k-1}$, so that $I_{k} \supsetneq I_{k-1}$. We also have

$$
c_{i}^{I_{k}}\left(x_{k-1}\right)=\sum_{j \in I_{k}} d_{j i}^{I_{k}}\left\langle x_{k-1}, \alpha_{j}\right\rangle=d_{l i}^{I_{k}}\left\langle x_{k-1}, \alpha_{l}\right\rangle
$$

Since $d_{l i}^{I_{k}} \geq 0$ for all $i$ and $d_{l l}^{I_{k}}>0$ by Lemma 2.1, conditions (1) and (2) of the algorithm are satisfied.

We point out that one can always use the $I_{k}$ described in the preceding paragraph and this is perhaps the easiest choice. In the algorithm, we have allowed some flexibility in the choice of $I_{k}$ mainly so that we will be able to recover some results in the literature (see Section 4).

Note that the algorithm terminates in $n$ or fewer steps since $I_{0} \subsetneq I_{1} \subsetneq I_{2} \subsetneq \cdots \subsetneq$ $I \subseteq N$

THEOREM 2.5. If $x \in E$, then there exist $I_{x} \subseteq N, x^{-} \in E^{-}\left(I_{x}\right)$, and $x^{+} \in E^{+}\left(I_{x}\right)$ such that $x=x^{-}+x^{+}$. Moreover, $I_{x}, x^{-}$, and $x^{+}$are uniquely determined by $x$.

Proof. Let $x \in E$. As pointed out, the above algorithm applied to $x$ terminates with, say, $x_{t}$. Then $\left(x_{t}, \alpha_{j}\right) \geq 0$ for all $j$. Moreover, by Lemma $2.3(1),\left(x_{t}, \alpha_{j}\right)=0$ for all $j \in I_{t}$. Thus $x_{t} \in E^{+}\left(I_{t}\right)$. For each $i$, we have

$$
\left(x-x_{t}, \lambda_{i}\right)=\left(\sum_{k=1}^{t}\left(x_{k-1}-x_{k}\right), \lambda_{i}\right)=\sum_{k=1}^{t}\left(x_{k-1}\left(I_{k}\right), \lambda_{i}\right) .
$$


If $i \notin I_{r}$, then $i \notin I_{k}$ for each $k$, whence $\left(x-x_{i}, \lambda_{i}\right)=0$ by Lemma 2.3 (2). On the other hand, if $i \in I_{t}=\cup_{k} I_{k}$, then $i \in I_{m} \backslash I_{m-1}$ for some $m$, so using the equation above and then Lemma 2.3 (2) and the choice of the $I_{k}$, we get

$$
\left(x-x_{t}, \lambda_{i}\right) \leq\left(x_{m-1}\left(I_{m}\right), \lambda_{i}\right)<0 .
$$

Also, it is easy to see that $x_{k-1}$ and $x_{k}$ have the same component in $E_{0}$ for each $k$, so $x-x_{t} \in E_{1}$. Therefore, $x-x_{t} \in E^{-}\left(I_{t}\right)$.

Setting $x^{+}=x_{t}, x^{-}=x-x_{t}$ and $I_{x}=I_{t}$, we have $x^{-} \in E^{-}\left(I_{x}\right), x^{+} \in E^{+}\left(I_{x}\right)$ and $x=x^{-}+x^{+}$, as desired.

To prove uniqueness, suppose we have $I, I^{\prime} \subseteq N, x^{ \pm} \in E^{ \pm}(I), x^{ \pm^{\prime}} \in E^{ \pm}\left(I^{\prime}\right)$, with $x^{-}+x^{+}=x=x^{-\prime}+x^{+^{\prime}}$. From Lemma 2.2 we get

$$
\sum_{i \in I} a_{i} \alpha_{i}+\sum_{j \notin l} b_{j} \lambda_{j}=\sum_{i \in I^{\prime}} a_{i}^{\prime} \alpha_{i}+\sum_{j \notin I^{\prime}} b_{j}^{\prime} \lambda_{j}
$$

with $a_{i}, a_{i}^{\prime}<0$ and $b_{j}, b_{j}^{\prime} \geq 0$ where the four sums are $x^{-}, x^{+}-x^{0}, x^{-^{\prime}}$, and $x^{+^{\prime}}-x^{0}$, respectively ( $x^{0}$ denoting the component of $x$ in $E_{0}$ ).

First assume $I \cap I^{\prime}=\phi$. Let $j \in I$ and apply $\left\langle\cdot, \alpha_{j}\right\rangle$ to both sides of $(*)$ to get

$$
\sum_{i \in I} a_{i}\left\langle\alpha_{i}, \alpha_{j}\right\rangle=c_{j},
$$

where $c_{j}=\sum_{i \in I^{\prime}} a_{i}^{\prime}\left\langle\alpha_{i}, \alpha_{j}\right\rangle+b_{j}^{\prime} \geq 0\left(\right.$ since $\left.\left\langle\alpha_{i}, \alpha_{j}\right\rangle \leq 0, i \neq j\right)$. It follows that

$$
a_{i}=\sum_{j \in I} d_{j i}^{l} c_{j}
$$

for all $i \in I$. By Lemma $2.1(1), d_{j i}^{\prime} \geq 0$ for each $i, j \in I$, so we get $a_{i} \geq 0$ for each $i \in I$, which is a contradiction unless $I=\phi$. Similarly, we get a contradiction unless $I^{\prime}=\phi$. Thus $I \cap I^{\prime}=\phi$ implies $I=\phi$ and $I^{\prime}=\phi$.

Now return to the general case and set $J=\left\{i \in I \cap I^{\prime}: a_{i}<a_{i}^{\prime}\right\}$ and $J^{\prime}=\{i \in$ $I \cap I^{\prime}: a_{i}>a_{i}^{\prime}$. By rearranging terms in $(*)$, we obtain

$$
\sum_{i \in K} e_{i} \alpha_{i}+\sum_{j \notin K} b_{j} \lambda_{j}=\sum_{i \in K^{\prime}} e_{i}^{\prime} \alpha_{i}+\sum_{j \notin K^{\prime}} b_{j}^{\prime} \lambda_{j},
$$

where $K=\left(I \backslash I^{\prime}\right) \cup J, K^{\prime}=\left(I^{\prime} \backslash I\right) \cup J^{\prime}$,

$$
e_{i}=\left\{\begin{array}{ll}
a_{i}, & i \in I \backslash I^{\prime} \\
a_{i}-a_{i}^{\prime}, & i \in J,
\end{array} \quad e_{i}^{\prime}= \begin{cases}a_{i}^{\prime}, & i \in I^{\prime} \backslash I \\
a_{i}^{\prime}-a_{i}, & i \in J^{\prime},\end{cases}\right.
$$

and where $b_{j}=0$ if $j \in I, b_{j}^{\prime}=0$ if $j \in I^{\prime}$. The previous paragraph applies to give $K=\phi=K^{\prime}$. This in turn implies $I=I^{\prime}$ and $a_{i}=a_{i}^{\prime}$ for all $i$. Hence $(*)$ becomes $\sum_{j \notin I} b_{j} \lambda_{j}=\sum_{j \notin I^{\prime}} b_{j}^{\prime} \lambda_{j}$. Since the set $\left\{\lambda_{j}: j \in N\right\}$ is linearly independent, we have $b_{j}=b_{j}^{\prime}$ for all $j$. Therefore, we have shown that $I=I^{\prime}, x^{-}=x^{-\prime}$ and $x^{+}=x^{+^{\prime}}$ and have thus completed the proof of uniqueness. 
For future reference, we record the following consequence of the proof of Theorem 2.5.

COROLlaRY 2.6. Let $x \in E$ and suppose Algorithm 2.4 applied to $x$ terminates with the element $x_{t}$ of $E$. Then $x^{+}=x_{t}, x^{-}=x-x_{t}$, and $I_{x}=I_{t}$. In particular, $x_{t}$ is independent of the choices made in the algorithm.

Set $E^{+}=E^{+}(\phi)=\left\{x \in E:\left(x, \alpha_{j}\right) \geq 0\right.$ for all $\left.j\right\}$. By Lemma 2.2, $x \in E^{+}$if and only if $x=x^{0}+\sum_{j} b_{j} \lambda_{j}$ with $x^{0} \in E_{0}, b_{j} \geq 0$. According to [H2, p. 22], $E^{+}$is a fundamental domain for the action of $W$ on $E$, that is, for each $x \in E$, the orbit $W x$ intersects $E^{+}$in precisely one point, which we denote by $\bar{x}$.

We obtain a partial order on $E$ by putting $x \prec y$ if $\left(y-x, \lambda_{j}\right) \geq 0$ for all $j$, or, equivalently, $y-x=\sum_{i} a_{i} \alpha_{i}$ with $a_{i} \geq 0$. For the proof of the following lemma, see [H2, p. 22].

LEMMA 2.7. If $x \in E$, then $x \prec \bar{x}$.

For the next lemma, we need a few notions, which we state here in sufficient generality to be applicable in the next section as well. Let $V$ be a real vector space. A subset $C$ of $V$ is convex if $t x+(1-t) y \in C$ for all $x, y \in C, 0 \leq t \leq 1$. If $X \subseteq V$, then the convex hull $C(X)$ of $X$ is the intersection of all convex subsets of $V$ containing $X$. It is easily seen that, if $X$ is finite, then $C(X)=\left\{\sum_{x \in X} a_{x} x: \sum a_{x}=1\right\}$. A function $\varphi: V \rightarrow \mathbb{R}$ is convex if $\varphi(t x+(1-t) y) \leq t \varphi(x)+(1-t) \varphi(y)$ for all $x, y \in V, 0 \leq t \leq 1$. If $V$ is acted on by a group $G$, then a function $\varphi: V \rightarrow \mathbb{R}$ is $G$-invariant if $\varphi(g x)=\varphi(x)$ for all $g \in G, x \in V$.

PROPOSITION 2.8. Let $x, y \in E$. The following are equivalent:

(1) $\bar{x}<\bar{y}$,

(2) $\varphi(x) \leq \varphi(y)$ for every $W$-invariant convex function $\varphi: E \rightarrow \mathbb{R}$,

(3) $x \in C(W y)$.

PROOF. The equivalence of (1) and (3) is proved in [Ko, Lemma 3.3] in the case $\Phi$ is a set of 'restricted' roots; the proof carries over to this setting. (2) and (3) are equivalent by $[\mathrm{AB}$, p. 599].

Given $x, y \in E$, define $y_{x}=x-(x-y)^{+}$and note that $y_{x}=y+(x-y)^{-}$as well, since $x-y=(x-y)^{-}+(x-y)^{+}$. Here, the superscripts + and - refer to the notation in Theorem 2.5. In view of Corollary 2.6, Algorithm 2.4 can be used to compute $y_{x}$.

PROPOSITION 2.9. If $x, y \in E^{+}$, then $y_{x}$ is the unique element of $E$ satisfying (1) $y_{x} \in C(W y) \cap E^{+}$, 
(2) $x-y_{x} \in E^{+}$, and

(3) $\left(x-y_{x}, y_{x}-y\right)=0$.

PROOF. Let $x, y \in E^{+}$. First we show that $y_{x}$ satisfies the three properties.

(1) If $j \in I:=I_{x-y}$, then $\left(y_{x}, \alpha_{j}\right)=\left(x, \alpha_{j}\right)-\left((x-y)^{+}, \alpha_{j}\right)=\left(x, \alpha_{j}\right) \geq 0$ since $x \in E^{+}$and $(x-y)^{+} \in E^{+}(I)$. Similarly, if $j \notin I$, then $\left(y_{x}, \alpha_{j}\right)=\left(y, \alpha_{j}\right)+$ $\left((x-y)^{-}, \alpha_{j}\right)=\left(y, \alpha_{j}\right)+\sum_{i \in I}\left((x-y)^{-}, \lambda_{i}\right)\left\langle\alpha_{j}, \alpha_{i}\right\rangle \geq 0$ where we have used that $\alpha_{j}=\sum_{i}\left\langle\alpha_{j}, \alpha_{i}\right\rangle \lambda_{i},(x-y)^{-} \in E^{-}(I), y \in E^{+}$and $\left\langle\alpha_{j}, \alpha_{i}\right\rangle \leq 0$ for $i \in I$. Therefore, $\left(y_{x}, \alpha_{j}\right) \geq 0$ for all $j$ implying $y_{x} \in E^{+}$.

For any $i$ we have $\left(y_{x}-y, \lambda_{i}\right)=\left((x-y)^{-}, \lambda_{i}\right) \leq 0$ since $(x-y)^{-} \in E^{-}(I)$. Thus $y_{x} \prec y$. From Proposition 2.8 and the previous paragraph, we conclude that $y_{x} \in C(W y)$.

(2) We have $x-y_{x}=(x-y)^{+} \in E^{+}(I) \subseteq E^{+}$.

(3) We have $\left(x-y_{x}, y_{x}-y\right)=\left((x-y)^{+},(x-y)^{-}\right)=0$ by Lemma 2.2 (3).

Now assume $z \in E$ satisfies the three properties (with $z$ in place of $y_{x}$ ). Since $z \in C(W y) \cap E^{+}$, Proposition 2.8 implies $z \prec y$. Therefore, $z-y=\sum_{i \in I} a_{i} \alpha_{i}$ for some $I \subseteq N$ and $a_{i}<0$, which is to say $z-y \in E^{-}(I)$ (Lemma 2.2 (1)). Since $x-z \in E^{+}$, we have $x-z=u^{0}+\sum_{j} b_{j} \lambda_{j}$ with $u^{0} \in E_{0}, b_{j} \geq 0$. Now

$$
\frac{1}{2} \sum_{i \in I} a_{i} b_{i}\left(\alpha_{i}, \alpha_{i}\right)=\sum_{i \in I, j \in N} a_{i} b_{i}\left(\lambda_{j}, \alpha_{i}\right)=(x-z, z-y)=0,
$$

where we have used that $\left(\lambda_{j}, \alpha_{i}\right)=\left\langle\lambda_{j}, \alpha_{i}\right\rangle\left(\alpha_{i}, \alpha_{i}\right) / 2=\delta_{i j}\left(\alpha_{i}, \alpha_{i}\right) / 2$. Since $\left(\alpha_{i}\right.$, $\left.\alpha_{i}\right)>0$ for each $i$, we conclude that $b_{j}=0$ for all $j \in I$. This says that $x-z \in E^{+}(I)$ (Lemma 2.2 (2)). Finally, $x-y=(z-y)+(x-z)$ so the uniqueness part of Theorem 2.5 says $x-z=(x-y)^{+}$, implying $z=x-(x-y)^{+}=y_{x}$.

LEMMA 2.10. If $x \in E$, then $x^{+} \in C(W x)$.

Proof. First, we observe that $x^{0} \in C(W x)$ for any $x \in E$, where $x^{0}$ is the component of $x$ in $E_{0}$. To see this, let $x \in E$. Then $\bar{x} \in E^{+}$, implying $\bar{x}=$ $\bar{x}^{0}+\sum_{j} b_{j} \lambda_{j}=\bar{x}^{0}+\sum_{i, j} b_{j} d_{j i} \alpha_{i}$, with $b_{j} \geq 0$ (Lemma $2.2(2)$ ). Since $d_{j i} \geq 0$ (Lemma 2.1 (1)), we have $\bar{x} \succ \bar{x}^{0}=\bar{x}^{0}$. Therefore, Proposition 2.8 gives $x^{0} \in C(W x)$.

Now to prove the lemma, let $x \in E$. If $j \in I:=I_{x}$ (notation as in Theorem 2.5), then $\left(x^{+}, \alpha_{j}\right)=0$ (since $\left.x^{+} \in E^{+}(I)\right)$, so $s_{\alpha_{j}}\left(x^{+}\right)=x^{+}-\left\langle x^{+}, \alpha_{j}\right\rangle \alpha_{j}=x^{+}$. It follows that $W_{l}$ fixes $x^{+}$. Therefore, $x^{+}=0+x^{+}=\left(x^{-}\right)^{0}+x^{+} \in C\left(W_{l} x^{-}\right)+x^{+}=$ $C\left(W_{l} x^{-}+x^{+}\right)=C\left(W_{l}\left(x^{-}+x^{+}\right)\right) \subseteq C(W x)$. (Here, $\left(x^{-}\right)^{0}$ is the component of $x^{-}$in the orthogonal complement in $E$ of the span of $\Phi_{l}$, so the previous paragraph applies to give $\left(x^{-}\right)^{0} \in C\left(W_{l} x^{-}\right)$.)

LEMMA 2.11. Let $x, y \in E$ and assume $x \prec y$. 
(1) If $y \in E^{+}$and $I \subseteq N$, then $x-x(I) \prec y$.

(2) $x^{+} \prec y^{+}$.

Proof. (1) Assume $y \in E^{+}$and let $i \in I \subseteq N$. From Lemma 2.3 (2), we have

$$
\left(x(I), \lambda_{i}\right)=c_{i} \sum_{k \in I} d_{k i}^{I}\left\langle x, \alpha_{k}\right\rangle,
$$

where $c_{i}=\left(\alpha_{i}, \alpha_{i}\right) / 2$. We can write $x-y$ in the form $x-y=\sum_{j} e_{j} \alpha_{j}$ and we find that $e_{j}=\left(x-y, \lambda_{j}\right) / c_{j} \leq 0$ by applying $\left(\cdot, \lambda_{j}\right)$ to both sides. Since $y \in E^{+}$and $d_{k i}^{I} \geq 0$ (Lemma $\left.2.1(1)\right)$, we get

$$
\begin{aligned}
\left(x(I), \lambda_{i}\right) & \geq c_{i} \sum_{k \in I} d_{k i}^{I}\left\langle x, \alpha_{k}\right\rangle-c_{i} \sum_{k \in I} d_{k i}^{I}\left\langle y, \alpha_{k}\right\rangle \\
& =c_{i} \sum_{k \in I} d_{k i}^{I}\left\langle x-y, \alpha_{k}\right\rangle=c_{i} \sum_{\substack{k \in I \\
j \in N}} e_{j} d_{k i}^{I}\left\langle\alpha_{j}, \alpha_{k}\right\rangle \\
& \geq c_{i} \sum_{j, k \in I} e_{j} d_{k i}^{I}\left\langle\alpha_{j}, \alpha_{k}\right\rangle=c_{i} \sum_{j \in I} e_{j} \delta_{i j}=\left(x-y, \lambda_{i}\right),
\end{aligned}
$$

where the second inequality is due to the fact that $\left\langle\alpha_{j}, \alpha_{k}\right\rangle \leq 0$ for $j \neq k$. Hence, $\left(x(I), \lambda_{i}\right) \geq\left(x-y, \lambda_{i}\right)$ for each $i \in I$. On the other hand, if $i \notin I$, then $\left(x(I), \lambda_{i}\right)=0$ by Lemma $2.3(2)$. We conclude that

$$
\left(x-x(I)-y, \lambda_{i}\right)=\left(x-y, \lambda_{i}\right)-\left(x(I), \lambda_{i}\right) \leq 0,
$$

for each $i$, which says that $x-x(I) \prec y$.

(2) Since $y^{-} \in E^{-}\left(I_{y}\right)$, we have $\left(y-y^{+}, \lambda_{i}\right)=\left(y^{-}, \lambda_{i}\right) \leq 0$ for each $i$. Hence $y \prec y^{+}$, implying $x \prec y^{+}$as well. Using the notation of Algorithm 2.4, we get from (1) that $x_{k} \prec y^{+}$for each $k$. By Corollary 2.6, $x^{+}$equals the terminal vector $x_{t}$ produced by the algorithm applied to $x$. Hence, $x^{+} \prec y^{+}$, as desired.

On $W$ is defined a length function (relative to $\Delta$ ). There is a unique element of maximal length, which we denote by $w_{0}$ (called the longest element of $W$ ) $[\mathrm{H} 2$, Section 1.8].

LEMMA 2.12. If $x, y \in E^{+}$and $z \in C(W y)$, then $x-y_{x} \prec \overline{x-z} \prec x-w_{0} y$ and $w_{0} y=-(\overline{-y})$.

Proof. Let $x, y \in E^{+}$and $z \in C(W y)$. By Proposition 2.8 and Lemma 2.7, we have $z \prec y$, implying $x-y \prec x-z$. Using Lemma 2.11(2), we get $x-y_{x}=(x-y)^{+} \prec$ $(x-z)^{+}=x-z_{x}$. But Lemma 2.10 implies $x-z_{x}=(x-z)^{+} \in C(W(x-z))$, so that $x-y_{x} \prec x-z_{x} \prec \overline{x-z_{x}} \prec \overline{x-z}$ by Lemma 2.7 and Proposition 2.8 . 
For the other inequality, we first remark that $\overline{a+b} \prec \bar{a}+\bar{b}$ for every $a, b \in E$. Indeed, if $a, b \in E$, then there exists $w \in W$ with $w(a+b)=\overline{a+b}$, so that $\overline{a+b}=w a+w b \prec \bar{a}+\bar{b}$ (Lemma 2.7). Next, since $z \in C(W y)$, we have $-z \in C(W(-y))$. Thus, $\overline{-z} \prec \overline{-y}$ by Proposition 2.8. Putting this together with the remark above, we get $\overline{x-z} \prec \bar{x}+\overline{-z} \prec \bar{x}+\overline{-y}$. Now $\overline{-y} \in W(-y)$, implying $-(\overline{-y}) \in W y \cap-E^{+}$. Since $W$ acts simply transitively on the Weyl chambers and $w_{0} E^{+}=-E^{+}\left[\mathrm{H} 2\right.$, Section 1.8], we conclude that $-(\overline{-y})=w_{0} y$. Hence, $\overline{x-z} \prec \bar{x}+\overline{-y}=\bar{x}-w_{0} y$.

Before stating the main result of the section, we remind the reader that, given $x, y \in E$, Algorithm 2.4 can be used to compute the element $y_{x}$ of $E$ (in at most $n=|\Delta|$ steps). More precisely, the algorithm applied to $x-y$ terminates with the element $x-y_{x}$ (see remarks before Proposition 2.9).

THEOREM 2.13. Let $x, y \in E$, write $x=w \bar{x}$ with $w \in W$, and let $\varphi: E \rightarrow \mathbb{R}$ be a convex $W$-invariant function.

(1) The set $\{\varphi(x-z): z \in C(W y)\}$ has minimum $\varphi\left(\bar{x}-\bar{y}_{\bar{x}}\right)$; this minimum is attained when $z=w \bar{y}_{\bar{x}}$.

(2) The set $\{\varphi(x-z): z \in C(W y)\}$ has maximum $\varphi\left(\bar{x}-w_{0} \bar{y}\right)$; this maximum is attained when $z=w w_{0} \bar{y}$.

PROOF. From the $W$-invariance of $\varphi$ and the $W$-stability of $C(W y)$ we get $\{\varphi(x-z)$ : $z \in C(W y)\}=\{\varphi(\bar{x}-z): z \in C(W y)\}$, so we may assume $x, y \in E^{+}$(so that $\bar{x}=x, \bar{y}=y$, and $w=1$ ). By Proposition 2.9, $x-y_{x} \in E^{+}$, and by Lemma 2.12, $x-w_{0} y=x+\overline{-y} \in E^{+}$, so Proposition 2.8 and Lemma 2.12 give $\varphi\left(x-y_{x}\right) \leq \varphi(x-z) \leq \varphi\left(x-w_{0} y\right)$ for every $z \in C(W y)$. Since $y_{x} \in C(W y)$ by Proposition 2.9, and obviously $w_{0} y \in C(W y)$, the result now follows.

In general, the minimum of $\{\varphi(x-z): z \in C(W y)\}$ can be attained at more than one $z \in C(W y)$ and this is true for the maximum as well. However, by slightly strengthening the assumptions on $\varphi$ we can obtain uniqueness in the case of the minimum. Let $V$ be a real vector space. A convex function $\varphi: V \rightarrow \mathbb{R}$ is strictly convex if $\varphi((x+y) / 2)<(\varphi(x)+\varphi(y)) / 2$ for all $x, y \in V$ with $\varphi(x)=\varphi(y)$ and $x \neq y$.

COROLlaRY 2.14. Let $x, y \in E$ and write $x=w \bar{x}$ with $w \in W$. If $\varphi: E \rightarrow \mathbb{R}$ is a strictly convex $W$-invariant function, then $w \bar{y}_{\bar{x}}$ is the unique element of $C(W y)$ for which

$$
\varphi\left(x-w \bar{y}_{\bar{x}}\right)=\min \{\varphi(x-z): z \in C(W y)\} .
$$

In particular, $w \bar{y}_{\bar{x}}$ is the unique element of $C(W y)$ for which the above equation holds for every convex W-invariant function $\varphi: E \rightarrow \mathbb{R}$. 
PROOF. Let $\varphi: E \rightarrow \mathbb{R}$ be a strictly convex $W$-invariant function. Assume $\varphi$ attains a minimum at both $a$ and $b$ in the convex set $A:=x-C(W y)$. Using the fact that $(a+b) / 2 \in A$ and then the convexity of $\varphi$, we get $\varphi(a) \leq \varphi((a+b) / 2) \leq$ $(\varphi(a)+\varphi(b)) / 2=\varphi(a)$ so that the inequalities are in fact equalities. Since $\varphi$ is strictly convex, we conclude that $a=b$. This, in conjunction with Theorem 2.13 , proves the first statement.

Let $\varphi$ be the norm on $E$ induced by the inner product: $\varphi(a)=\sqrt{(a, a)}$. Then $\varphi$ is $W$ invariant (since $W$ is generated by reflections, which are orthogonal transformations), $\varphi$ is convex (as is obviously any norm), and $\varphi$ is strictly convex (by the parallelogram law). Therefore, the second statement follows from the first and Theorem 2.13.

\section{Extension to reductive Lie groups}

We begin this section with a discussion of those parts of the theory of reductive Lie groups that will be needed to state and prove our results. The definition of 'reductive Lie group' varies from author to author. For us, a reductive Lie group will be a member of the so-called Harish-Chandra class, which we now describe (see [Kn, p. 384]).

DEFINITION 3.1. The Harish-Chandra class $\mathscr{H}$ consists of 4-tuples $(G, K, \theta, B)$, where $G$ is a Lie group, $K$ is a compact subgroup of $G, \theta$ is a Lie algebra involution of the Lie algebra $\mathfrak{g}$ of $G$, and $B$ is a nondegenerate, $\operatorname{Ad}(G)$-invariant, symmetric, bilinear form on $\mathfrak{g}$ such that

(1) $\mathfrak{g}$ is reductive (meaning, $\mathfrak{g}=\mathfrak{g}_{1} \dot{\mathfrak{z}}$, where $\mathfrak{g}_{1}=[\mathfrak{g}, \mathfrak{g}]$ and $\mathfrak{z}$ is the center of $\mathfrak{g}$ ),

(2) $\mathfrak{g}=\mathfrak{k}+\mathfrak{p}$ (called the Cartan decomposition), where $\mathfrak{k}=$ Lie $K$ is the +1 eigenspace and $\mathfrak{p}$ is the -1 -eigenspace under the action of $\theta$,

(3) $\mathfrak{k}$ and $\mathfrak{p}$ are orthogonal with respect to $B$, and $B$ is negative definite on $\mathfrak{k}$ and positive definite on $\mathfrak{p}$,

(4) the map $K \times \exp p \rightarrow G$ given by multiplication is a surjective diffeomorphism,

(5) for every $g \in G$, the automorphism $\operatorname{Ad}(g)$ of $\mathfrak{g}$, extended to the complexification $\mathfrak{g}^{\mathrm{C}}$ of $\mathfrak{g}$ is contained in Int $\mathfrak{g}^{\mathrm{C}}$, and

(6) the analytic subgroup $G_{1}$ of $G$ with Lie algebra $\mathfrak{g}_{1}=[\mathfrak{g}, \mathfrak{g}]$ has finite center.

If $(G, K, \theta, B) \in \mathscr{H}$, then $G$ is called a reductive Lie group.

Note that (5) is automatically satisfied if $G$ is connected, for then $\operatorname{Ad}(G)=\operatorname{Int} \mathfrak{g} \subseteq$ Int $g^{C}$.

EXAMPLE 3.2. Let $G$ be a (connected) semisimple Lie group with finite center, let $B$ be the Killing form on $\mathfrak{g}=$ Lie $G$, let $\theta: \mathfrak{g} \rightarrow \mathfrak{g}$ be a Lie algebra involution such that the form $B_{\theta}(x, y):=-B(x, \theta(y))$ is positive definite (called a Cartan involution), let 
$\mathfrak{g}=\mathfrak{k}+\mathfrak{p}$ be as in Definition 3.1(2), and let $K$ be the analytic sur: If with L: algebra $k^{k}$. Then $(G, K, \theta, B) \in \mathscr{H}[\mathrm{Kn}, \mathrm{p}$. 385].

EXAMPLE 3.3. Let $G$ be a closed linear group of real or cony utrices closed under conjugate transpose inverse $\left(X \mapsto\left(X^{*}\right)^{-1}\right)$, given as th: of some set of real-valued polynomials in the real and imaginar; entries, and satisfying Definition 3.1 (5). Let $\theta$ be negative $c$ $\left(x \mapsto-x^{*}\right)$, let $K$ be the intersection of $G$ with the unitary group, $\operatorname{Re} \operatorname{Tr}(x y)$. Then $(G, K, \theta, B) \in \mathscr{H}[\mathrm{Kn}, \mathrm{p} .385]$.

For the remainder of this section, we fix $(G, K, \theta, B) \in \mathscr{H}$ at of Definition 3.1.

Among the Abelian subalgebras of $\mathfrak{g}$ that are contained in $\mathfrak{p}$, chc $\mathfrak{a}$ (referred to as a maximal Abelian subspace of $\mathfrak{p}$ ). For $\alpha \in \mathfrak{a}^{*}(=$ on zero lucu: of the mat: $i x$ te trans ose let $B(x, y)=$ set

$$
\mathfrak{g}_{\alpha}=\{x \in \mathfrak{g}:[h, x]=\alpha(h) x \text { for all } h \in \mathfrak{a}\} .
$$

If $0 \neq \alpha \in \mathfrak{a}^{*}$ and $\mathfrak{g}_{\alpha} \neq 0$, then $\alpha$ is called a (restricted) root of the 1 air $(\mathfrak{g}, \mathfrak{a})$. The set of roots will be denoted $\Sigma$. We have $\mathfrak{g}=\mathfrak{g}_{0} \dot{+} \dot{\sum}_{\alpha \in \Sigma} \mathfrak{g}_{\alpha}$.

We view $\mathfrak{a}$ as a Euclidean space by taking the inner product to be the restriction of $B$ to $\mathfrak{a}$. The map $\mathfrak{a}^{*} \rightarrow \mathfrak{a}$ that assigns to each $\lambda \in \mathfrak{a}^{*}$ the unique ement $x_{\lambda}$ of $\mathfrak{a}$ satisfying $\lambda(x)=B\left(x, x_{\lambda}\right)$ for all $x \in \mathfrak{a}$ is a vector space isomorphisr . We use this isomorphism to identify $\mathfrak{a}^{*}$ with $\mathfrak{a}$, allowing us, in particular, to view $\Sigma$ as a subset of a. The set $\Phi=\left\{\alpha \in \Sigma: \frac{1}{2} \alpha \notin \Sigma\right\}$ is a root system in $\mathfrak{a}$ in the sense of Section 2, called the reduced root system of the pair $(\mathfrak{g}, \mathfrak{a})$. Its associated finite reflection group $W$ is called the Weyl group. Clearly, $W$ is generated by the reflections $s_{\alpha}(\alpha \in \Sigma)$. As in Section 2, fix a base $\Delta$ for the root system $\Phi$. Then $\Delta$ determines a fundamental domain $\mathfrak{a}^{+}\left(=E^{+}\right.$of Section 2$)$ for the action of $W$ on $\mathfrak{a}$.

We now describe another way to view the Weyl group $W$. Use juxtaposition to represent the adjoint action of $G$ on $\mathfrak{g}: g x=\operatorname{Ad}(g)(x)(g \in G, x \in \mathfrak{g})$. Set $N_{K}(\mathfrak{a})=\{k \in K: k \mathfrak{a} \subseteq \mathfrak{a}\}$ and $Z_{K}(\mathfrak{a})=\{k \in K: k x=x$ for all $x \in \mathfrak{a}\}$. Then the action of $K$ on $\mathfrak{g}$ induces an action of the group $N_{K}(\mathfrak{a}) / Z_{K}(\mathfrak{a})$ on $\mathfrak{a}$. There exists an isomorphism $\psi: W \rightarrow N_{K}(\mathfrak{a}) / Z_{K}(\mathfrak{a})$ that is compatible with the two actions on $\mathfrak{a}$, or more precisely, for which $w x=\psi(w) x(w \in W, x \in \mathfrak{a})[\mathrm{Kn}, 7.32]$. We use the isomorphism $\psi$ to identify these two groups. Note in particular that, given $x \in \mathfrak{a}$, we have $W x=N_{K}(\mathfrak{a}) x \subseteq K x$.

The following result is well known for the case of semisimple $G$. For the reader's convenience, we supply the short proof in our more general setting.

LEMMA 3.4. If $x \in \mathfrak{p}$, then $\left|K x \cap \mathfrak{a}^{+}\right|=1$. 
ProOF. Let $x \in \mathfrak{p}$. Since $\mathfrak{p}=K \mathfrak{a}$ [Kn, 7.29], there exists some $k \in K$ for which $k x \in \mathfrak{a}$. Suppose also $k^{\prime} x \in \mathfrak{a}\left(k^{\prime} \in K\right)$ and write $k x=a, k^{\prime} x=a^{\prime}\left(a, a^{\prime} \in \mathfrak{a}\right)$. Exponentiating the equation $k^{-1} a=x=k^{\prime-1} a^{\prime}$ gives $k^{-1}(\exp a) k=k^{-1}\left(\exp a^{\prime}\right) k^{\prime}$ [Kn, 1.90]. According to [Kn, 7.39] we then have $\exp a^{\prime}=n(\exp a) n^{-1}=\exp (n a)$ for some $n \in W$. Since the exponential map on $\mathfrak{a}$ is injective [Kn, 1.104, 7.31], we conclude that $a^{\prime}=n a$. Thus, we have shown that $K x \cap \mathfrak{a}=W a$. Hence, $\left|K x \cap \mathfrak{a}^{+}\right|=\left|W a \cap \mathfrak{a}^{+}\right|=|\{\bar{a}\}|=1$.

Given $x \in \mathfrak{p}$, we denote the unique element of $K x \cap \mathfrak{a}^{+}$(which Lemma 3.4 guarantees) by $\bar{x}$. If $x \in \mathfrak{a}$, then the proof of Lemma 3.4 shows that $\bar{x}$ is the unique element of $W x \cap \mathfrak{a}^{+}$, so this notation is consistent with that in Section 2.

We will require two classical results from the theory of Lie groups-one due to Kostant and the other due to Berezin and Gel'fand. These are both statements about semisimple Lie groups and hence not general enough to be immediately applicable to our situation. Therefore, we provide extensions to the case of a reductive Lie group. (See Theorem 3.6 and Theorem 3.9.)

Let $\mathfrak{a}_{\perp}$ denote the orthogonal complement in $\mathfrak{p}$ of $\mathfrak{a}$ and let $\pi: \mathfrak{p} \rightarrow \mathfrak{a}$ denote the orthogonal projection of $\mathfrak{p}$ onto $\mathfrak{a}$ (where orthogonality is relative to the form $B$ ). As a consequence of the next lemma, $\pi$ is independent of the choice of $B$ (provided, of course, $B$ satisfies Definition 3.1).

LEMMA 3.5. $\mathfrak{a}_{\perp}=[\mathfrak{k}, \mathfrak{a}]$.

ProOF. First, we remark that, since $B$ is $\operatorname{Ad}(G)$ - invariant, it is ad( $(\mathfrak{g})$-invariant as well, which means $B([x, y], z)=-B(y,[x, z])(x, y, z \in \mathfrak{g})$. Indeed, for any $t \in \mathbb{R}$ we have $B(\operatorname{Ad}(\exp t x)(y), z)=B\left(y, \operatorname{Ad}(\exp t x)^{-1}(z)\right)=B(y, \operatorname{Ad}(\exp (-t x))(z))$, so differentiating and putting $t=0$ gives the indicated identity (see [Kn, p. 55]).

Let $x \in \mathfrak{k}, y \in \mathfrak{a}$. For any $z \in \mathfrak{a}$, we have $B([x, y], z)=B(x,[y, z])=B(x, 0)=$ 0 , since $\mathfrak{a}$ is Abelian. Since $[x, y] \in \mathfrak{p}$ (Definition $3.1(2)$ ), we have $[x, y] \in \mathfrak{a}_{\perp}$. Thus, $[\mathfrak{k}, \mathfrak{a}] \subseteq \mathfrak{a}_{\perp}$.

Let $\alpha \in \Sigma$ and let $x \in \mathfrak{g}_{\alpha}$. First, $x+\theta x \in \mathfrak{k}$ (see Definition 3.1 (2)). Choose $h_{\alpha} \in \mathfrak{a}$ with $\alpha\left(h_{\alpha}\right) \neq 0$. According to [Kn, 6.40 (c)], $\theta x \in \mathfrak{g}_{-\alpha}$ (the result is stated for semisimple $\mathfrak{g}$, but the proof is valid for reductive $\mathfrak{g}$, as well), so $y_{x, \alpha}:=$ $\alpha\left(h_{\alpha}\right) x-\alpha\left(h_{\alpha}\right) \theta x=\left[h_{\alpha}, x+\theta x\right] \in[\mathfrak{k}, \mathfrak{a}]$.

For each $\alpha \in \Sigma$, let $B_{\alpha}$ be a basis for $\mathfrak{g}_{\alpha}$. The set $\left\{y_{x, \alpha}: \alpha \in \Sigma^{+}, x \in B_{\alpha}\right\}$ is clearly linearly independent (where $\Sigma^{+}$is the set of those nonnegative linear combinations of $\Delta$ that are contained in $\Sigma$ ). Hence, $\operatorname{dim}[\mathfrak{k}, \mathfrak{a}] \geq \sum_{\alpha \in \Sigma^{+}} \operatorname{dim} \mathfrak{g}_{\alpha}$.

On the other hand, the Iwasawa decomposition gives $\mathfrak{g}=\mathfrak{k} \dot{+} \mathfrak{a} \dot{+} \mathfrak{n}$, where $\mathfrak{n}=$ $\sum_{\alpha \in \Sigma^{+}} \mathfrak{g}_{\alpha}$, from which it follows that $\operatorname{dim} \mathfrak{a}_{\perp}=\operatorname{dim} \mathfrak{n}=\sum_{\alpha \in \Sigma^{+}} \operatorname{dim} \mathfrak{g}_{\alpha}$. Therefore, $[\mathfrak{k}, \mathfrak{a}]=\mathfrak{a}_{\perp}$, as desired. 
The next result is commonly referred to as the 'Convexity Theorem'.

THEOREM 3.6 (Kostant). If $x \in \mathfrak{p}$, then $\pi(K x)=C(W \bar{x})$.

Proof. First assume $G$ is semisimple. By Lemma 3.5, $\pi$ is independent of the choice of $B$, so we may assume $B$ is the Killing form on $\mathfrak{g}$ (Example 3.3 and [Kn, p. 386]). In this case, the theorem is the well-known result proved by Kostant in [Ko].

Now let $G$ be arbitrary once again and let $x \in \mathfrak{p}$. Since $K x=K \bar{x}$, we may assume $x \in \mathfrak{a}$. The centralizer in $K$ of a meets every connected component of $K$ [Kn, 7.33], so denoting this centralizer by $M$, we have $K=K^{0} M$, where $K^{0}$ is the connected component of $K$ containing the identity element. Therefore, $K x=K^{0} M x=K^{0} x$.

Write $\mathfrak{k}_{1}, \mathfrak{p}_{1}, \mathfrak{a}_{1}$ (respectively, $\mathfrak{k}_{0}, \mathfrak{p}_{0}, \mathfrak{a}_{0}$ ) for the intersections of $\mathfrak{k}, \mathfrak{p}, \mathfrak{a}$ with $\mathfrak{g}_{1}$ (respectively, $\mathfrak{z}$ ) (notation as in Definition 3.1). Using [Kn, 7.19e, Example 1 on p. 385], we see that $\left(G_{1}, K_{1}, \theta_{1}, B_{1}\right) \in \mathscr{H}$, where $G_{1}$ is as in Definition 3.1, $K_{1}=\exp \left(\mathfrak{k}_{1}\right)$, and $\theta_{1}$ and $B_{1}$ are the restrictions to $\mathfrak{g}_{1}$ of $\theta$ and $B$, respectively. The Cartan decomposition of $\mathfrak{g}_{1}$ determined by $\theta_{1}$ is $\mathfrak{g}_{1}=\mathfrak{k}_{1} \dot{+} \mathfrak{p}_{1}$ and $\mathfrak{a}_{1}$ is a maximal Abelian subspace of $\mathfrak{p}_{1}\left[\mathrm{Kn}, \mathrm{p}\right.$. 393]. We have $\mathfrak{k}=\mathfrak{k}_{0} \dot{+} \mathfrak{k}_{1}, \mathfrak{p}=\mathfrak{p}_{0} \dot{+} \mathfrak{p}_{1}, \mathfrak{a}=\mathfrak{a}_{0} \dot{+} \mathfrak{a}_{1}$, and $\mathfrak{a}_{0}=\mathfrak{p}_{0}[\mathrm{Kn}, 7.28]$. By $[\mathrm{Kn}, 4.48], K^{0}=\exp \mathfrak{k}=\exp \mathfrak{k}_{1} \exp \mathfrak{k}_{0}$. Since $\left(\exp \mathfrak{k}_{0}\right) x=x$ [Kn, 1.93], we have $K^{0} x=K_{1} x$.

Now $[\mathfrak{k}, \mathfrak{a}]=\left[\mathfrak{k}_{1}, \mathfrak{a}_{1}\right]$, and so it follows from Lemma 3.5 that, for any $y=y_{0}+y_{1} \in \mathfrak{p}$ $\left(y_{i} \in \mathfrak{p}_{i}\right)$, we have $\pi(y)=y_{0}+\pi_{1}\left(y_{1}\right)$, where $\pi_{1}$ is the orthogonal projection of $\mathfrak{p}_{1}$ onto $\mathfrak{a}_{1}$ relative to $B_{1}$. Write $x=x_{0}+x_{1}$ with $x_{i} \in \mathfrak{a}_{i}$. According to [Kn, p. 394], $W x=x_{0}+W_{1} x_{1}$, where $W_{1}$ is the Weyl group associated with the reduced root system of the pair $\left(\mathfrak{g}_{1}, \mathfrak{a}_{1}\right)$.

Assembling these results and using the special case of the theorem discussed in the first paragraph, we obtain

$$
\begin{aligned}
\pi(K x) & =\pi\left(K_{1} x\right)=\pi\left(x_{0}+K_{1} x_{1}\right)=x_{0}+\pi_{1}\left(K_{1} x_{1}\right) \\
& =x_{0}+C\left(W_{1} x_{1}\right)=C\left(x_{0}+W_{1} x_{1}\right)=C(W x)=C(W x),
\end{aligned}
$$

as desired.

Next, we generalize a theorem of Raïs [R] (which was rediscovered by Lewis [L, Theorem 4.3]).

THEOREM 3.7 (Raïs). A $K$-invariant function $\varphi: \mathfrak{p} \rightarrow \mathbb{B}$ is convex if and only if its restriction to $a$ is convex.

PROOF. That the first condition implies the second is clear. Now let $\varphi: \mathfrak{p} \rightarrow \mathbb{R}$ be a $K$-invariant function and assume $\left.\varphi\right|_{\mathfrak{a}}$ is convex. Let $x, y \in \mathfrak{p}, 0 \leq t \leq 1$, and set $z=t x+(1-t) y$. Since $\varphi$ is $K$ - invariant, we may assume that $z \in \mathfrak{a}$. We have 
$\varphi(z)=\varphi(\pi(z))=\varphi(t \pi(x)+(1-t) \pi(x)) \leq t \varphi(\pi(x))+(1-t) \varphi(\pi(y))$. Now $x \in K x$, so Theorem 3.6 says $\pi(x) \in C(W \bar{x})$. Moreover, since $\varphi$ is $K$-invariant, $\left.\varphi\right|_{a}$ is $W$-invariant, so Proposition 2.8 applies to give $\varphi(\pi(x)) \leq \varphi(\bar{x})=\varphi(x)$. Similarly, $\varphi(\pi(y)) \leq \varphi(y)$. Hence $\varphi(z) \leq t \varphi(x)+(1-t) \varphi(x)$, as desired.

From this theorem of Raïs, we easily obtain a result that generalizes a theorem of Li and Tsing on unitary similarity invariant norms [LT1], as well as a theorem of von Neumann on unitarily invariant norms [vN]. (See Example 4.1 and Example 4.3.)

THEOREM 3.8. Let $\varphi: \mathfrak{p} \rightarrow \mathbb{R}$ be a function. Then $\varphi$ is a $K$-invariant norm if and only if there exists a W-invariant norm $\psi: \mathfrak{a} \rightarrow \mathbb{R}$ such that $\varphi(x)=\psi(\bar{x})(x \in \mathfrak{p})$.

Proof. First suppose $\varphi$ is a $K$-invariant norm and set $\psi=\left.\varphi\right|_{a}$. Then $\psi$ is clearly a $W$-invariant norm and for any $x \in \mathfrak{p}, \varphi(x)=\varphi(\bar{x})=\psi(\bar{x})$.

To prove the converse, suppose there exists a $W$-invariant norm $\psi: \mathfrak{a} \rightarrow \mathbb{R}$ such that $\varphi(x)=\psi(\bar{x})(x \in \mathfrak{p})$. Then $\varphi$ is clearly $K$-invariant, so it remains to be shown that $\varphi$ is a norm. For any $x \in \mathfrak{p}$, we have $\varphi(x)=\psi(\bar{x}) \geq 0$ and $\varphi(x)=0$ if and only if $\psi(\bar{x})=0$ if and only if $\bar{x}=0$ if and only if $x=0$. Next, let $x \in \mathfrak{p}$, $0 \neq r \in \mathbb{R}$. Then $\overline{r x}=k r x=r k x$ for some $k \in K$. Since $k x=r^{-1} \overline{r x} \in \mathfrak{a}$, we have $\varphi(r x)=\psi(\overline{r x})=\psi(r k x)=|r| \psi(k x)=|r| \psi(\bar{x})=|r| \varphi(x)$. Finally, by Theorem $3.7, \varphi$ is convex, so (using the previous step) $\frac{1}{2} \varphi(x+y)=\varphi\left(\frac{1}{2} x+\frac{1}{2} y\right) \leq \frac{1}{2} \varphi(x)+\frac{1}{2} \varphi(y)$ for any $x, y \in \mathfrak{p}$, and the triangle inequality follows.

Now we generalize to the case of a reductive Lie group the other classical result we require.

THEOREM 3.9 (Berezin-Gel'fand). If $x, y \in \mathfrak{p}$, then $\overline{x+y} \in \bar{x}+C(W \bar{y})$.

ProOF. First assume $G$ is semisimple. Then the theorem follows from [BGe, Theorem 3, p. 235]. Indeed, that theorem says $\overline{x+y}=\overline{(1, x+y)}=\overline{(1, x)(1, y)} \epsilon$ $C(\bar{x}+W \bar{y})=\bar{x}+C(W \bar{y})(x, y \in \mathfrak{p})$, where the pairs are viewed as elements of $\tilde{G}=K \times \mathfrak{p}$, and $\mathfrak{p}$ is viewed as a subset of $\tilde{G}$ via the injection $x \mapsto(1, x)(x \in \mathfrak{p})$.

Now assume $G$ is arbitrary once again and let the notation be as in the proof of Theorem 3.6. We will make a series of observations that will allow us to easily reduce to the special case above. First note that for any $x, y \in \mathfrak{g}$ and $z \in \mathfrak{z}$, the $\operatorname{ad}(\mathfrak{g})$-invariance of $B$ (see proof of Lemma 3.5) gives

$$
B([x, y], z)=B(x,[y, z])=B(x, 0)=0,
$$

so that $\mathfrak{g}_{1}$ is orthogonal to $\mathfrak{z}$ relative to $B$. Hence, $\mathfrak{a}=\mathfrak{a}_{0} \dot{+} \mathfrak{a}_{1}$ is an orthogonal direct sum. 
Next, it is easy to see that any root of the pair $(\mathfrak{g}, \mathfrak{a})$ is zero on $\mathfrak{a}_{0}$ and that restriction to $\mathfrak{a}_{1}$ maps $\Phi$ bijectively onto the reduced root system $\Phi_{1}$ of the pair $\left(\mathfrak{g}_{1}, \mathfrak{a}_{1}\right)[\mathrm{Kn}$, p. 393]. Recall that we identify $\mathfrak{a}^{*}$ with $\mathfrak{a}$ by mapping $\lambda \in \mathfrak{a}^{*}$ to the unique $x_{\lambda} \in \mathfrak{a}$ for which $\lambda=B\left(x_{\lambda}, \cdot\right)$, and hence view $\Phi$ as a subset of $\mathfrak{a}$. For any $\alpha \in \Phi, x \in \mathfrak{a}_{0}$, we have $B\left(x_{\alpha}, x\right)=\alpha(x)=0$, implying $\Phi$ is contained in the orthogonal complement in $\mathfrak{a}$ of $\mathfrak{a}_{0}$, which is $\mathfrak{a}_{1}$ according to the previous paragraph. An easily seen consequence of this is that $\Phi$ is precisely the copy of $\Phi_{1}$ in $\mathfrak{a}_{1}$ (the copy being given by the identification $a_{1}^{*} \rightarrow a_{1}$ induced by $B_{1}$ ).

The Weyl group $W$ fixes $\mathfrak{a}_{0}$ elementwise (since $\Phi$ is orthogonal to $\mathfrak{a}_{0}$ ), and restriction to $a_{1}$ defines an isomorphism $W \rightarrow W_{1}$ where $W_{1}$ is the Weyl group associated with $\Phi_{1}$. We clearly have $\mathfrak{a}^{+}=\mathfrak{a}_{0}+\mathfrak{a}_{1}^{+}$, where $\mathfrak{a}_{1}^{+}$is the fundamental domain for the action of $W_{1}$ on $a_{1}$ corresponding to the base $\Delta_{1}:=\Delta$ of $\Phi_{1}$.

Let $z \in \mathfrak{p}$. By [Kn, 7.29], $z \in K_{1}$ a. Using this, together with the remarks above about the Weyl groups, we find that $\bar{z}=k z=z_{0}+k z_{1}$ for some $k \in K_{1}$. Hence $k z_{1}=\bar{z}-z_{0} \in \mathfrak{a}^{+} \cap \mathfrak{p}_{1}=\mathfrak{a}_{1}^{+}$, implying $k z_{1}=\bar{z}_{1}$, where $\bar{z}_{1}$ is the representative in $\mathfrak{a}_{1}^{+}$ of the $K_{1}$-orbit of $z_{1}$. We conclude that $\bar{z}=z_{0}+\bar{z}_{1}$.

Now we can finish the proof by using the special case of the first paragraph. For any $x, y \in \mathfrak{p}$, we have $\overline{x+y}=x_{0}+y_{0}+\overline{x_{1}+y_{1}} \in x_{0}+y_{0}+\overline{x_{1}}+C\left(W_{1} \overline{y_{1}}\right)=$ $\bar{x}+C\left(y_{0}+W_{1} \overline{y_{1}}\right)=\bar{x}+C\left(W\left(y_{0}+\overline{y_{1}}\right)\right)=\bar{x}+C(W \bar{y})$.

The second part of the following corollary was proved in [T] for the case of semisimple $G$.

COROLlaRY 3.10. Let $x, y \in \mathfrak{p}$.

(1) $\overline{\bar{x}-\bar{y}} \prec \overline{x-y}$.

(2) $\overline{x+y} \prec \bar{x}+\bar{y}$.

Proof. (1) Using Theorem 3.9, we have $\bar{x}=\overline{y+(x-y)} \in \bar{y}+C(W \overline{x-y})$. Therefore, $\bar{x}-\bar{y} \in C(W \overline{x-y})$ and the result follows from Proposition 2.8 .

(2) Using Lemma 2.7 and then (1), we obtain $\overline{x+y}-\bar{y} \prec \overline{\overline{x+y}-\bar{y}} \prec \bar{x}$. The inequality follows.

REMARK. The inequalities in the corollary can be viewed as generalizations of the classical triangle inequalities of real analysis: ||$x|-| y|| \leq|x-y|$ and $|x+y| \leq$ $|x|+|y|(x, y \in \mathbb{R})$. Indeed, if $G=S L(2, \mathbb{C}), K=S U(2), \theta: x \mapsto-x^{*}$, $B=$ Killing form, $\mathfrak{a}=\left\{\left[\begin{array}{cc}r & 0 \\ 0 & -r\end{array}\right]: r \in \mathbb{R}\right\}$, then $\Phi$ is irreducible of type $A_{1}$ in $\mathfrak{a}$, which identifies with $\mathbb{R}$. The Weyl group consists of the identity map and negation, so if we choose $\mathfrak{a}^{+}=\mathbb{R}^{+}$, then $\bar{x}=|x|$ and also $x \prec y$ if and only if $x \leq y$ $(x, y \in \mathfrak{a})$. Thus, for any $x, y \in \mathfrak{a} \subseteq \mathfrak{p}$, we can apply the corollary to obtain the triangle inequalities. 
We will require the following easy corollary of Theorem 3.6. Given $X \subseteq \mathfrak{p}$, we denote by $\bar{X}$ the set $\{\bar{x}: x \in X\}$.

COROLlaRY 3.11. If $x \in \mathfrak{a}$, then

$$
\overline{C(K x)}=C(K x) \cap \mathfrak{a}^{+}=C(W x) \cap \mathfrak{a}^{+}=\overline{C(W x)} .
$$

PRoof. Let $x \in \mathfrak{a}$. We have

$$
\begin{aligned}
\overline{C(K x)} & \subseteq C(K x) \cap \mathfrak{a}^{+} & & \text {(using the } K \text {-stability of } C(K x) \text { ) } \\
& \subseteq \pi(C(K x)) \cap \mathfrak{a}^{+} & & \text {(since } \pi \text { fixes a pointwise) } \\
& =C(\pi(K x)) \cap \mathfrak{a}^{+} & & \text {(since } \pi \text { is linear) } \\
& =C(W x) \cap \mathfrak{a}^{+} & & \text {(by Theorem 3.6) } \\
& \subseteq \overline{C(W x)} & & \\
& \subseteq \overline{C(K x)} & & \text { (since } W x \subseteq K x) .
\end{aligned}
$$

Since the first and last expressions are the same, the containments must be equalities.

We are now in a position to prove an analog of Theorem 2.13 in our reductive Lie group setting. In the statement of the theorem, $\bar{y}_{\bar{x}}$ has the same meaning as in Section 2 (definition before Proposition 2.9) with $E=\mathfrak{a}, E^{+}=\mathfrak{a}^{+}$, and so forth, and $w_{0}$ is the longest element of the Weyl group $W$. (See also the comments before the statement of Theorem 2.13.)

THEOREM 3.12. Let $x, y \in \mathfrak{p}$, write $x=k \bar{x}$ with $k \in K$, and let $\varphi: \mathfrak{p} \rightarrow \mathbb{R}$ be a convex $K$-invariant function.

(1) The set $\{\varphi(x-z): z \in C(K y)\}$ has minimum $\varphi\left(\bar{x}-\bar{y}_{\bar{x}}\right)$; this minimum is attained when $z=k \bar{y}_{\bar{x}}$.

(2) The set $\{\varphi(x-z): z \in C(K y)\}$ has maximum $\varphi\left(\bar{x}-w_{0} \bar{y}\right)$; this maximum is attained when $z=k w_{0} \bar{y}$.

PROOF. As in the proof of Theorem 2.13, we may assume $x, y \in \mathfrak{a}^{+}$(so that $\bar{x}=x$, $\bar{y}=y$, and $k=1$ ). We have

$$
\begin{aligned}
& \min \{\varphi(x-z): z \in C(K y)\} \\
& =\min \{\varphi(\overline{x-z}): z \in C(K y)\} \quad \text { (using } K \text {-invariance of } \varphi \text { ) } \\
& \geq \min \{\varphi(x-\bar{z}): z \in C(K y)\} \quad \text { (Corollary } 3.10 \text { (1) and Proposition 2.8) } \\
& =\min \{\varphi(x-z): z \in \overline{C(K y)}\} \quad \text { (straightforward) } \\
& \geq \min \{\varphi(x-z): z \in C(W y)\} \quad \text { (Corollary 3.11). }
\end{aligned}
$$


Similarly, we have

$$
\begin{aligned}
\max & \{\varphi(x-z): z \in C(K y)\} \\
& =\max \{\varphi(\overline{x-z}): z \in C(K y)\} \\
& \leq \max \{\varphi(x+\overline{-z}): z \in C(K y)\} \quad \text { (Corollary 3.10(2) and Proposition 2.8) } \\
& =\max \{\varphi(x+z): z \in \overline{-C(K y)}\} \\
& \leq \max \{\varphi(x-z): z \in C(W y)\} .
\end{aligned}
$$

On the other hand, $\{\varphi(x-z): z \in C(K y)\} \supseteq\{\varphi(x-z): z \in C(W y)\}$ (since $K y \supseteq W y$ ), so the inequalities above are in fact equalities. The theorem now follows from Theorem 2.13.

COROLlARY 3.13. Let $x, y \in \mathfrak{p}$ and write $x=k \bar{x}$ with $k \in K$. If $\varphi: \mathfrak{p} \rightarrow \mathbb{R}$ is a strictly convex $K$-invariant function, then $k \bar{y}_{\bar{x}}$ is the unique element of $C(K y)$ for which

$$
\varphi\left(x-k \bar{y}_{\bar{x}}\right)=\min \{\varphi(x-z): z \in C(K y)\} .
$$

In particular, $k \bar{y}_{\bar{x}}$ is the unique element of $C(K y)$ for which the above equation holds for every convex $K$-invariant function $\varphi: \mathfrak{p} \rightarrow \mathbb{R}$.

PROOF. The proof of the first statement is the same as that for the corresponding statement in Corollary 2.14. The norm $\varphi$ on $\mathfrak{p}$ induced by the inner product $B$ is $K$-invariant by the $\operatorname{Ad}(G)$ - invariance of $B$, so the second statement also follows as before.

Finally, we apply some of our results to a useful special case. Let $U$ be a compact connected Lie group with Lie algebra $\mathfrak{u}$. We can view $U$ as a subgroup of some unitary group $U(n)<G L(n, \mathbb{C})$ by the Peter-Weyl theorem, and in turn view $u$ as a subalgebra of $\mathfrak{g l}(n, \mathbb{C})$. Let $\mathfrak{u}^{\mathbb{C}}=\mathfrak{u} \dot{+} i \mathfrak{u}(i=\sqrt{-1})$ be the complexification of $\mathfrak{u}$ and let $U^{\mathbb{C}}$ be the analytic subgroup of $G L(n, \mathbb{C})$ with Lie algebra $\mathfrak{u}^{\mathbb{C}}$. Define $\theta: \mathfrak{u}^{\mathbb{C}} \rightarrow \mathfrak{u}^{\mathbb{C}}$ by $x+i y \mapsto x-i y(x, y \in \mathfrak{u})$. Let $B$ be the Killing form on $\mathfrak{u}$ extended to $\mathfrak{u}^{\mathrm{C}}$ by setting $B(x+i y, u+i v)=B(x, u)-B(y, v)$. Then $\left(U^{\mathrm{C}}, U, \theta, B\right) \in \mathscr{H}[\mathrm{Kn}$, p. 404, Examples 1 and 3 on p. 385, Remark on p. 380]. The corresponding Cartan decomposition of $\mathfrak{u}^{\mathbb{C}}$ is $\mathfrak{u}^{\mathfrak{C}}=\mathfrak{k}+\mathfrak{p}$, where $\mathfrak{k}=\mathfrak{u}$ and $\mathfrak{p}=i \mathfrak{u}$. If $\mathfrak{t}$ is a maximal Abelian subalgebra of $\mathfrak{u}$, then $\mathfrak{a}=i$ is a maximal Abelian subspace of $\mathfrak{p}$. Clearly, $\mathfrak{u}$ and $\mathfrak{p}$ are isomorphic as modules for $K=U$. The following result can now be easily verified.

COROLlARY 3.14. The statements Theorem 3.7, Theorem 3.8, Theorem 3.12, and Corollary 3.13 remain valid if $K, \mathfrak{p}, \mathfrak{a}$ are replaced by $U, \mathfrak{u}, \mathfrak{t}$, respectively.

The version of Theorem 3.7 given in the corollary was proved by Atiyah and Bott in [AB, Proposition 12.16]. 


\section{Examples}

In this final section, we show how our general results can be used to obtain results of Li and Tsing [LT2] and results of Cheng [C] that involve a set $\mathscr{M}$ of matrices, an equivalence relation $\sim$ on $\mathscr{M}$, and a $\sim$-invariant norm $\|\cdot\|$ on $\mathscr{M}$. For various choices of $\mathscr{M}$ and $\sim$, these authors solved the problem of finding, for fixed $X, Y \in \mathscr{M}$, the extreme values of $\|X-Z\|$ as $Z$ ranges through the convex hull of the equivalence class of $Y$. Here we take their choices for $\mathscr{M}$ and $\sim$ one at a time and show that in each case, there exists $(G, K, \theta, B) \in \mathscr{H}$ such that (in the notation of Definition 3.1) $\mathscr{M}=\mathfrak{p}$ and $\sim$ is given by congruence under the adjoint action of $K$. (Actually, in Example 4.4 we need to use a group slightly larger than $K$ in order to obtain the stated equivalence relation, and in the last Example 4.6, we use instead the adjoint action of a compact Lie group on its Lie algebra (which plays the role of $\mathscr{M}$ ) as in Corollary 3.14). Since $\|\cdot\|$ is convex and $K$-invariant, our main result, Theorem 3.12 , then applies to give the aforementioned extreme values. We show that our findings are in agreement with those in the literature.

Besides the one on extreme values, there are other results in the papers of $\mathrm{Li}$ and Tsing and by Cheng that can be recovered by using our general results. We discuss a few of these in the first example and leave to the interested reader the similar verifications in the remaining examples.

For each example (save the last), we specify $G$ and $K$, and take $\theta$ to be negative conjugate transpose and $B(x, y)=c \operatorname{Re} \operatorname{Tr}(x y)$ (c, an appropriate real constant) appealing to Example 3.3 for justification that $(G, K, \theta, B)$ is in $\mathscr{H}$. The adjoint action of $K$ on $\mathfrak{p}$ in each case is given by $\operatorname{Ad}(k)(x)=k x k^{-1}(k \in K, x \in \mathfrak{p})$.

EXAMPLE 4.1. $\mathscr{M}$ is the set of $n \times n$ Hermitian matrices and $\sim$ is unitary similarity: $X \sim Y$ if and only if $X=U Y U^{*}$ for some $U \in U(n)$ (cf. [LT2], [C, type (I), p. 170]).

Let $G=G L(n, \mathbb{C})$ and $K=U(n)$. We have $\mathfrak{g}=\mathfrak{g l}(n, \mathbb{C}), \mathfrak{k}=\mathfrak{u}(n)(=$ algebra of skew Hermitian matrices), and $\mathfrak{p}=$ set of $n \times n$ Hermitian matrices. We may take as maximal Abelian subspace $\mathfrak{a}$ of $\mathfrak{p}$ the set of $n \times n$ real diagonal matrices, which we identify in the obvious way with $\mathbb{R}^{n}=\left\{x=\left(x_{1}, \ldots, x_{n}\right): x_{i} \in \mathbb{R}\right\}$. Let $B$ be given by $B(x, y)=\operatorname{Re} \operatorname{Tr}(x y)$. Then restriction of $B$ to a yields the standard inner product: $B(x, y)=\sum_{i} x_{i} y_{i}(x, y \in \mathfrak{a})$. The (reduced) root system $\Phi$ of the pair $(\mathfrak{g}, \mathfrak{a})$ is irreducible of type $A_{n-1}$. Viewed as a subset of $\mathfrak{a}$, as usual, $\Phi$ spans $\{x \in \mathfrak{a}$ : $\left.\sum_{i} x_{i}=0\right\}$, which has as orthogonal complement the line $\{(c, \ldots, c): c \in \mathbb{R}\}$. For a base $\Delta$ of $\Phi$, we may take the set $\left\{\alpha_{1}, \ldots, \alpha_{n-1}\right\}$, where $\alpha_{i}=e_{i}-e_{i+1}\left(\left\{e_{1}, \ldots, e_{n}\right\}\right.$ being the standard basis of $\left.\mathbb{R}^{n}\right)$. The Weyl group $W$ associated with $\Phi$ identifies with the symmetric group $\Sigma_{n}$ on $\{1, \ldots, n\}$ via $\sigma x=\left(x_{\sigma(1)}, \ldots, x_{\sigma(n)}\right)(\sigma$ in $W$ on the left and in $\Sigma_{n}$ on the right, $x \in \mathfrak{a}$ ). The fundamental domain for the action of $W$ on $\mathfrak{a}$ 
determined by $\Delta$ is $\mathfrak{a}^{+}=\left\{x \in \mathfrak{a}: x_{1} \geq \cdots \geq x_{n}\right\}$.

First, we apply Theorem 3.8 in this setting to recover Theorem 4.1 of $\mathrm{Li}$ and Tsing in [LT1]. Let $H_{n}$ denote the set of $n \times n$ Hermitian matrices. A norm $\|\cdot\|$ on $H_{n}$ is unitary similarity invariant if $\left\|U A U^{-1}\right\|=\|A\|$ for all $A \in H_{n}, U \in U(n)$. A function $\Phi: \mathbb{R}^{n} \rightarrow \mathbb{R}$ is Schur convex if $\Phi(x) \leq \Phi(y)$ whenever $x \in C\left(\Sigma_{n} y\right)$, where the action of $\Sigma_{n}$ on $\mathbb{R}^{n}$ is as described above. Li and Tsing's result states that, for every unitary similarity invariant norm $\|\cdot\|$ on $H_{n}$, there exists a Schur convex norm $\Phi$ on $\mathbb{R}^{n}$ such that $\|A\|=\Phi(\lambda(A))$ for each $A \in H_{n}$, where $\lambda(A)$ is the $n$-tuple of eigenvalues of $H$ arranged in nonincreasing order. In the notation of this example, we have $\mathfrak{p}=H_{n}$, and a unitary similarity invariant norm on $\mathfrak{p}$ is the same as a $K$ invariant norm. By Proposition 2.8, a $W$-invariant norm on $\mathbb{R}^{n}$ is a Schur convex norm. Therefore, $\mathrm{Li}$ and Tsing's result follows from Theorem 3.8 after we note that $\lambda(A)=\bar{A}\left(A \in H_{n}\right)$.

Next, using the fact that the longest element $w_{0}$ of the Weyl group sends $\alpha_{i}$ to $-\alpha_{n-i}$ and fixes $(1, \ldots, 1)$, one easily checks that $w_{0} x=\left(x_{n}, \ldots, x_{1}\right)(x \in \mathfrak{a})$. Therefore, Theorem 1 in [LT2] follows from Theorem 3.12 above.

Now we consider Theorem 3 of [LT2]. Our aim is to show that, for suitable choices of the sets $I_{k}$, Algorithm 2.4 coincides with the algorithm of Li and Tsing. Let $x \in \mathfrak{a}$ (corresponding to an arbitrary choice of $\Delta \in \mathbb{R}^{n}$ in their Step 1). To avoid confusion with coordinate notation, we denote the element of $\mathfrak{a}$ obtained in the $r$ th step of our algorithm by $x^{(r)}$ instead of $x_{r}$.

First, observe that our algorithm ends with $x^{(r)}$ if and only if $x_{k}^{(r)}-x_{k-1}^{(r)}=$ $\left(x^{(r)}, \alpha_{k}\right) \geq 0$ for all $k$, the same as in Li and Tsing's algorithm. Suppose our algorithm does not end with $x^{(r-1)}$. Then $x_{k-1}^{(r-1)}-x_{k}^{(r-1)}=\left(x^{(r-1)}, \alpha_{k-1}\right)<0$ for some $k>1$. Let $1 \leq j<k \leq l \leq n$ be such that

$$
x_{j-1}^{(r-1)} \neq x_{j}^{(r-1)}=\cdots=x_{k-1}^{(r-1)}<x_{k}^{(r-1)}=\cdots=x_{l}^{(r-1)} \neq x_{l+1}^{(r-1)},
$$

where the first (respectively, last) member is ignored if $j=1$ (respectively, $l=n$ ).

Set $I_{r}=I_{r-1} \cup J$, where $J=\{j, \ldots, l-1\}$. We need to show that $I_{r}$ satisfies the two conditions of Algorithm 2.4. If $b \in M:=I_{r} \backslash J$, then $b \in I_{r-1}$, so $\left(x^{(r-1)}, \alpha_{b}\right)=0$ by Lemma 2.3 (1), implying $b \neq j-1, l$. It follows that $\left(\alpha_{i}, \alpha_{b}\right)=0$ for $i \in J, b \in M$. Since $I_{r}=J \cup M$ (disjoint union), we get $d_{b i}^{l_{r}}=0$ if $(b, i) \notin(M \times M) \cup(J \times J)$. Therefore,

$$
c_{i}^{I_{r}}\left(x^{(r-1)}\right)=\sum_{b \in I_{r}} d_{b i}^{I_{r}}\left\langle x^{(r-1)}, \alpha_{b}\right\rangle=\sum_{b \in J} d_{b i}^{I_{r}}\left\langle x^{(r-1)}, \alpha_{b}\right\rangle=d_{k-1, i}^{J}\left\langle x^{(r-1)}, \alpha_{k-1}\right\rangle
$$

which, according to Lemma 2.1 and the choice of $k$, is nonpositive for every $i$ and negative for $i \in I_{r} \backslash I_{r-1}$ (since then $i, k-1 \in J \in \mathscr{L}$ ). This shows that the two conditions are satisfied. 
From the preceding paragraph, we get

$$
x^{(r)}=x^{(r-1)}-x^{(r-1)}\left(I_{r}\right)=x^{(r-1)}-\sum_{a \in J} d_{k-1, a}^{J}\left\langle x^{(r-1)}, \alpha_{k-1}\right\rangle \alpha_{a} .
$$

If $i<j$ or $i>l$, then $\left(\alpha_{a}, e_{i}\right)=0$ for all $a \in J$, implying $x_{i}^{(r)}=\left(x^{(r)}, e_{i}\right)=$ $\left(x^{(r-1)}, e_{i}\right)=x_{i}^{(r-1)}$. Next, $\left\{\alpha_{a}: a \in J\right\}$ is a base for a root system isomorphic to the irreducible root system of type $A_{l-j}$. By consulting [H1, p. 69], we find that $d_{k-1, l-1}^{J}=(k-j) /(l-j+1)$. Therefore,

$$
\begin{aligned}
x_{l}^{(r)} & =\left(x^{(r)}, e_{l}\right)=x_{l}^{(r-1)}+d_{k-1, l-1}^{J}\left\langle x^{(r-1)}, \alpha_{k-1}\right\rangle \\
& =x_{l}^{(r-1)}+\frac{k-j}{l-j+1}\left(x_{k-1}^{(r-1)}-x_{k}^{(r-1)}\right) \\
& =\frac{1}{l-j+1}\left[(k-j) x_{k-1}^{(r-1)}+(l-k+1) x_{k}^{(r-1)}\right]=\frac{1}{l-j+1} \sum_{b=j}^{l} x_{b}^{(r-1)},
\end{aligned}
$$

where we have used that $x_{b}^{(r-1)}=x_{k-1}^{(r-1)}$ for $j \leq b<k$, and $x_{b}^{(r-1)}=x_{k}^{(r-1)}$ for $k \leq b \leq l$. Now, if $j \leq i<l$, then $x_{i}^{(r)}-x_{i+1}^{(r)}=\left(x^{(r)}, \alpha_{i}\right)=0$ (Lemma 2.3 (1)), that is, $x_{i}^{(r)}=x_{i+1}^{(r)}$. Putting this together with the last computation, we get $x_{i}^{(r)}=(l-j+1)^{-1} \sum_{b=j}^{l} x_{b}^{(r-1)}$ for $j \leq i \leq l$. We showed earlier that $x_{i}^{(r)}=x_{i}^{(r-1)}$ for $i<j$ or $i>l$, so this finishes the proof that our algorithm coincides with that of $\mathrm{Li}$ and Tsing.

The reader should now have no difficulty in verifying that Theorem 3 of [LT2] follows from Theorem 3.12 (1) above. (Setting $x=A$ and $y=B$, we have $\bar{x}=\lambda(A)$, $k=U, k \bar{y}_{\bar{x}}=B_{m}$. Note that the algorithm applied to $\bar{x}-\bar{y}$ terminates with $(\bar{x}-\bar{y})^{+}=\bar{x}-\bar{y}_{\bar{x}}$ according to Corollary 2.6.)

Next, we show how Theorem 2 in [LT2] can be proved using Proposition 2.9 above. For this, we need the following observation.

Let $u \in \mathfrak{a}^{+}, v \in \mathfrak{a}$ and assume $v \succ 0$. Then $(u, v)=0$ if and only if there does not exist $1 \leq k<n$ such that $\sum_{i=1}^{k} v_{i}>0$ and $u_{k}>u_{k+1}$.

PROOF. Since $v \succ 0$, we have $v=\sum_{k=1}^{n-1} a_{k} \alpha_{k}=\sum_{k=1}^{n-1} a_{k}\left(e_{k}-e_{k+1}\right)$ with $a_{k} \geq 0$. Hence, for $1 \leq i \leq n$, we have $v_{i}=\left(a_{i}-a_{i-1}\right)$ (defining $a_{0}=0, a_{n}=0$ ). Thus, $\sum_{i=1}^{k} v_{i}=\sum_{i=1}^{k}\left(a_{i}-a_{i-1}\right)=a_{k} \geq 0(1 \leq k \leq n)$. Also, since $u \in \mathfrak{a}^{+}$, we have $u_{k}-u_{k+1} \geq 0(1 \leq k<n)$. Now $(u, v)=\sum_{i} u_{i} v_{i}=\sum_{k=1}^{n-1}\left[\left(u_{k}-u_{k+1}\right)\left(\sum_{i=1}^{k} v_{i}\right)\right]$, where the second equality uses the fact that $\sum_{i=1}^{n} v_{i}=a_{n}=0$. Therefore, the claim follows.

We now claim that Proposition 2.9 implies the following statement.

$$
\text { If } x, y \in \mathfrak{a}^{+} \text {and } z \in \mathfrak{p} \text {, then } z=y_{x} \text { if and only if }
$$


(1) $z \in C(K y) \cap \mathfrak{a}^{+}$,

(2) $x_{k}-z_{k} \geq x_{k+1}-z_{k+1}(1 \leq k<n)$, and

(3) there does not exist $1 \leq k<n$ such that $\sum_{i=1}^{k} y_{i}>\sum_{i=1}^{k} z_{i}$ and $x_{k}-z_{k}>$ $x_{k+1}-z_{k+1}$.

Proof. We intend to apply Proposition 2.9 with $E=\mathfrak{a}, E^{+}=\mathfrak{a}^{+}$, etc., as in the discussion before Lemma 3.4. First, Corollary 3.11 says $C(K y) \cap \mathfrak{a}^{+}=C(W y) \cap \mathfrak{a}^{+}$, so (1) agrees with Proposition 2.9(1). Next, we have already observed that $u \in \mathfrak{a}^{+}$if and only if $u_{k} \geq u_{k+1}(1 \leq k<n)$, so (2) agrees with Proposition 2.9(2). Finally, assuming (1) and (2), we have $z \prec y$ (Proposition 2.8) and $x-z \in \mathfrak{a}^{+}$, so the previous statement applies with $v=y-z$ and $u=x-z$ to finish the proof.

Now, with the aid of Corollary 3.13 concerning uniqueness in the case of a strictly convex function (which $\|\cdot\|_{2}$ is), it is not hard to see that the above statement is equivalent to Theorem 2 in [LT2] (with $x=A, y=B, z=B^{\prime}, y_{x}=\hat{B}$ ). (Actually, we get only the special case of that theorem with $U=I$, but the general case follows immediately.)

Finally, we indicate how the key Lemma 1 of [LT2] follows from our results. First, the set $\mathbb{R}_{\downarrow}^{n}$ is the same as our $\mathfrak{a}^{+}$. Next, $\mathrm{Li}$ and Tsing write $x \prec y\left(x, y \in \mathbb{R}^{n}\right)$ to mean that $x$ is 'majorized' by $y$. It is well known [MO] that this is equivalent to saying $x \in C\left(\Sigma_{n} y\right)=C(W y)$. Now $\Delta$ in the lemma is the terminal element of the algorithm applied to $x-y$, which is $(x-y)^{+}=x-y_{x}$ (Corollary 2.6), whence $y^{\prime}=y_{x}$. Therefore, parts (a) and (b) of the lemma follow from Proposition 2.9 (1), part (c) from Proposition 2.9 (2), and part (d) from Lemma 2.10.

EXAMPLE 4.2. $\mathscr{M}$ is the set of symmetric $n \times n$ matrices over $\mathbb{R}$ and $\sim$ is orthogonal similarity: $X \sim Y$ if and only if $X=O Y O^{t}$ for some $O \in O(n)$ (cf. [C, type (II), p. 170]).

Let $G=G L(n, \mathbb{R})$ and $K=O(n)$. We have $\mathfrak{g}=\mathfrak{g l}(n, \mathbb{R}), \mathfrak{k}=\mathbf{s o}(n)$ (= algebra of $n \times n$ skew symmetric matrices over $\mathbb{R}$ ), and $\mathfrak{p}=$ set of symmetric $n \times n$ matrices over $\mathbb{R}$. The discussion in Example 4.1 now carries over verbatim to this setting. (Cheng already pointed out in [C] that the results in [LT2] are valid for the $\mathscr{M}$ and $\sim$ of this example.)

EXAMPLE 4.3. $\mathscr{M}$ is the set $\mathbb{C}_{m \times n}$ of $m \times n$ matrices over $\mathbb{C}$ and $\sim$ is unitary equivalence: $X \sim Y$ if and only if $X=U Y V$ for some $U \in U(m), V \in U(n)$ (cf. [C, type (III), $\mathbb{F}=\mathbb{C}$, p. 171]).

Let $G=U(m, n)=\left\{g \in G L(m+n, \mathbb{C}): g^{*} I_{m, n} g=I_{m, n}\right\}$, where $I_{m, n}=\left[\begin{array}{cc}I_{m} & 0 \\ 0 & -I_{n}\end{array}\right]$ $\left(I_{t}=t \times t\right.$ identity matrix). Let $K=\left\{\left[\begin{array}{ll}U & 0 \\ 0 & v\end{array}\right]: U \in U(m), V \in U(n)\right\}$. (Since the unitary group is connected, so is $K$, and hence so is $G$ by [Kn, 1.122]. Therefore Definition 3.1 (5) is satisfied.) We have $\mathfrak{g}=\mathfrak{u}(m, n)=\left\{\left[\begin{array}{ll}a & b \\ b^{*} & d\end{array}\right]: a \in \mathfrak{u}(m), d \in\right.$ 
$\left.\mathfrak{u}(n), b \in \mathbb{C}_{m \times n}\right\}, \mathfrak{k}$ consists of those matrices in $\mathfrak{g}$ with $b=0$ and $\mathfrak{p}$ those with $a=0=d$ (see [Kn, p. 314]). We identify $p$ with $\mathbb{C}_{m \times n}$ via $\left[\begin{array}{cc}0 & b \\ b^{*} & 0\end{array}\right] \mapsto b$. With this identification, the adjoint action of $k=\left[\begin{array}{ll}U & 0 \\ 0 & V\end{array}\right] \in K$ on $b$ becomes $\operatorname{Ad}(k)(b)=U b V^{*}$, so the corresponding equivalence relation is unitary equivalence, as desired.

For the remainder of the discussion, we assume $m \geq n$ (the other case being similar). We may take $a=\left\{x=\sum_{i=1}^{n} x_{i} e_{i i}: a_{i} \in \mathbb{R}\right\} \subseteq \mathbb{C}_{m \times n}$ which we identify with $\mathbb{R}^{n}$ via $x \mapsto\left(x_{1}, \ldots, x_{n}\right)$. Let $B$ be given by $B(x, y)=\frac{1}{2} \operatorname{Re} \operatorname{Tr}(x y)$. Then restriction of $B$ to $\mathfrak{a}$ yields the standard inner product on $\mathbb{R}^{n}$. The reduced root system $\Phi$ of the pair $(\mathfrak{g}, \mathfrak{a})$ is the irreducible root system of type $B_{n}$ if $m>n$ and of type $C_{n}$ if $m=n$. We may take $\Delta=\left\{\alpha_{1}, \ldots, \alpha_{n}\right\}$, where $\alpha_{i}=e_{i}-e_{i+1}(1 \leq i<n)$ and $\alpha_{n}$ is $e_{n}$ or $2 e_{n}$ according as $m>n$ or $m=n$. Then $\mathrm{a}^{+}=\left\{x \in \mathfrak{a}: x_{1} \geq \cdots \geq x_{n} \geq 0\right\}$.

The Weyl group $W$ associated with $\Phi$ consists of all mappings $\mathfrak{a} \rightarrow \mathfrak{a}$ of the form $x \mapsto\left(\epsilon_{1} x_{\sigma(1)}, \ldots, \epsilon_{n} x_{\sigma(n)}\right)$, with $\epsilon_{i}= \pm 1$ and $\sigma \in \Sigma_{n}$, so the symmetric gauge function $\varphi: \mathfrak{a} \rightarrow \mathbb{R}$ referred to by Cheng is $W$-invariant (and convex since it is a norm).

Arguing as in Example 4.1, we see from Theorem 3.8 that a function $\|\cdot\|: \mathbb{C}_{m \times n} \rightarrow$ $\mathbb{R}$ is a norm and is constant on equivalence classes relative to $\sim$ (a so-called unitarily invariant norm) if and only if there exists a symmetric gauge function $\Phi$ on $\mathbb{R}^{n}$ such that $\|X\|=\Phi(s(X))$ for all $X \in \mathbb{C}_{m \times n}$, where $s(X)$ is the $n$-tuple of singular values of $X$ arranged in nonincreasing order. This is a theorem of von Neumann [vN].

The longest element $w_{0}$ of the Weyl group is negation, so Theorem $2(\mathrm{a})(\mathbb{F}=\mathbb{C})$ of [C] follows from Theorem 3.12 (2). The other results of [C] in the setting of this example that are analogs of the results of $\mathrm{Li}$ and Tsing discussed in Example 4.1 are obtained similarly. We comment only on the algorithm in the case $m>n$ (the case $m=n$ being similar).

Since the vectors $\alpha_{1}, \ldots, \alpha_{n-1}$ are the same as those in Example 4.1, we can apply the algorithm just as in that example to produce from $x \in \mathfrak{a}$ the vector $x^{(r)}$ with $x_{1}^{(r)} \geq \cdots \geq x_{n}^{(r)}$. This corresponds to repeating Step 3 of Theorem 1 in [C] up to the point where the algorithm passes to Step 4 .

If $x_{n}^{(r)}=\left(x^{(r)}, \alpha_{n}\right) \geq 0$, then our algorithm stops, as does Cheng's. Assume otherwise and let $k$ be the least index for which $x_{k}^{(r)}<0$. Let $J=\{k, \ldots, n\}$ and set $I_{r+1}=I_{r} \cup J$. Arguing as in Example 4.1, we get for any $i \in I_{r+1}$,

$$
c_{i}^{I_{r+1}}\left(x^{(r)}\right)=\sum_{j \in I_{r+1}} d_{j i}^{I_{r+1}}\left\langle x^{(r)}, \alpha_{j}\right\rangle=\sum_{j \in J} d_{j i}^{I_{r+1}}\left\langle x^{(r)}, \alpha_{j}\right\rangle,
$$

and if $i \in I_{r+1} \backslash J$, then $d_{j i}^{l_{r+1}}=0$ for all $j \in J$. If $i \in J$, then

$$
d_{j i}^{I_{+}+1}=d_{j i}^{J}= \begin{cases}i-k+1, & j<n, j \geq i \\ j-k+1, & j<n, j<i \\ (i-k+1) / 2, & j=n\end{cases}
$$


[H1, p. 69], and a straightforward computation gives $c_{i}^{l_{r+1}}\left(x^{(r)}\right)=\sum_{j=k}^{i} x_{j}<0$. Therefore, conditions (1) and (2) of Algorithm 2.4 are met. The terminal element of the algorithm is $x^{(r+1)} ;$ it satisfies $x_{i}^{(r+1)}=\left(x^{(r+1)}, e_{i}\right)=\left(x^{(r)}, e_{i}\right)=x_{i}^{(r)}$ for all $i \notin J$, while $x_{i}^{(r+1)}-x_{i+1}^{(r+1)}=\left(x^{(r+1)}, \alpha_{i}\right)=0(k \leq i<n)$ and $x_{n}^{(r+1)}=\left(x^{(r+1)}, \alpha_{n}\right)=0$, implying $x_{k}^{(r+1)}=x_{k+1}^{(r+1)}=\cdots=x_{n}^{(r+1)}=0$ just as in Step 4 of Cheng's algorithm. This shows, in light of our earlier remarks, that Theorem 1 of $[\mathrm{C}]$ is a special case of Theorem 2.13.

EXAMPLE 4.4. $\mathscr{M}$ is the set $\mathbb{R}_{m \times n}$ of $m \times n$ matrices over $\mathbb{R}$ and $\sim$ is orthogonal equivalence: $X \sim Y$ if and only if $X=U Y V$ for some $U \in O(m), V \in O(n)$ (cf. $[\mathrm{C}$, type (III), $\mathbb{F}=\mathbb{R}$, p. 171]).

It would be nice just to let $G=O(m, n)=\left\{g \in G L(m+n, \mathbb{R}): g^{t} I_{m, n} g=I_{m, n}\right\}$ and proceed in a manner analogous to Example 4.3. Unfortunately though, $O(m, n)$ does not always satisfy Definition 3.1(5) (for instance, when $m=n$ ). Therefore, we instead let $G=S O(m, n)^{\circ}$ (= identity component of the group $S O(m, n)$ of those elements of $O(m, n)$ having determinant 1). Set $K=\left\{\left[\begin{array}{ll}U & 0 \\ 0 & v\end{array}\right]: U \in S O(m), V \in\right.$ $S O(n)\}$. Then $\mathfrak{g}, \mathfrak{k}, \mathfrak{p}$, and $\mathfrak{a}$ are the intersections with $\mathfrak{g l}(m+n, \mathbb{R})$ of the corresponding spaces in Example 4.3. Assume $m \geq n$ (the case $m \leq n$ being similar). If $m>n$, then the reduced root system $\Phi$ of the pair $(\mathfrak{g}, \mathfrak{a})$ is $\Phi=\left\{ \pm e_{i} \pm e_{j}: i \neq j\right\} \cup\left\{ \pm e_{i}\right\}$ (signs read independently here and below), which is of type $B_{n}$, and taking $\Delta=$ $\left\{e_{i}-e_{i+1}(1 \leq i<n), e_{n}\right\}$ we have $\mathfrak{a}^{+}=\left\{x \in \mathfrak{a}: x_{1} \geq \cdots \geq x_{n} \geq 0\right\}$. If $m=n$, then $\Phi=\left\{ \pm e_{i} \pm e_{j}: i \neq j\right\}$, which is of type $D_{n}$, and taking $\Delta=\left\{e_{i}-e_{i+1}(1 \leq i<\right.$ $\left.n), e_{n-1}+e_{n}\right\}$, we have $\mathfrak{a}^{+}=\left\{x \in \mathfrak{a}: x_{1} \geq \cdots \geq x_{n-1} \geq\left|x_{n}\right|\right\}$.

Let $K^{\prime}=\left\{\left[\begin{array}{ll}U & 0 \\ 0 & v\end{array}\right]: U \in O(m), V \in O(n)\right\}$. Then $K^{\prime}=K D$, where $D$ is the set of diagonal matrices with diagonal entries \pm 1 . Let $K^{\prime}$ act on $\mathfrak{p}$ by conjugation, thus extending the action of $K$. Under this action, $D$ stabilizes $\mathfrak{a}$ and identifies with the group of reflections in $\mathfrak{a}$ of the form $x \mapsto\left(\epsilon_{1} x_{1}, \ldots, \epsilon_{n} x_{n}\right)\left(\epsilon_{i}= \pm 1\right)$. Clearly, the group $W^{\prime}$ generated by $W$ and $D$ is the finite reflection group associated with the root system $\Phi^{\prime}=\left\{ \pm e_{i} \pm e_{j}: i \neq j\right\} \cup\left\{ \pm e_{i}\right\}$ which is of type $B_{n}$. The set $\Delta^{\prime}=\left\{e_{i}-e_{i+1}(1 \leq i<n), e_{n}\right\}$ is a base for $\Phi^{\prime}$ and the corresponding fundamental domain for the action of $W^{\prime}$ on $\mathfrak{a}$ is $\mathfrak{a}^{+^{\prime}}=\left\{x \in \mathfrak{a}: x_{1} \geq \cdots \geq x_{n} \geq 0\right\}$.

Given $x \in \mathfrak{p}$, it is easy to see that the orbit $K^{\prime} x$ intersects $\mathfrak{a}^{+^{\prime}}$ in a unique element, which we write as $\bar{x}^{\prime}$. From Lemma 2.7 , we have $\bar{x} \prec^{\prime} \bar{x}^{\prime}(x \in \mathfrak{a})$, where $\prec^{\prime}$ is the partial order on $\mathfrak{a}$ induced by $\Delta^{\prime}$.

We claim that Theorem 3.12 is valid with $K, \bar{x}, \bar{y}, w_{0}$ replaced by $K^{\prime}, \bar{x}^{\prime}, \bar{y}^{\prime}, w_{0}^{\prime}$ (= longest element of $W^{\prime}$ ), and with $\bar{y}_{\bar{x}^{\prime}}^{\prime}$ computed relative to $\Delta^{\prime}$. Indeed, assuming $x, y \in \mathfrak{a}^{+\prime}$, we have, as in the proof of Theorem 3.12,

$$
\begin{aligned}
\min \left\{\varphi(x-z): z \in C\left(K^{\prime} y\right)\right\} & =\min \left\{\varphi(\overline{x-z}): z \in C\left(K^{\prime} y\right)\right\} \\
& \geq \min \left\{\varphi\left(x-\bar{z}^{\prime}\right): z \in C\left(K^{\prime} y\right)\right\}
\end{aligned}
$$




$$
=\min \left\{\varphi(x-z): z \in{\overline{C\left(K^{\prime} y\right)}}^{\prime}\right\},
$$

where the arguments are as before except with the additional observation that $\varphi(x-$ $\left.\bar{z}^{\prime}\right) \leq \varphi\left(x-\bar{z}\right.$ ) since $x-\bar{z}^{\prime} \prec^{\prime} x-\bar{z}$ (see Proposition 2.8). Now using Theorem 3.6, we obtain

$$
\pi\left(K^{\prime} y\right)=\pi\left(\bigcup_{d \in D} K d y\right)=\bigcup_{d \in D} \pi(K d y) \subseteq \bigcup_{d \in D} C(W \overline{d y}) \subseteq C\left(W^{\prime} y\right),
$$

so the argument in the proof of Corollary 3.11 applies to give ${\overline{C\left(K^{\prime} y\right)}}^{\prime} \subseteq C\left(W^{\prime} y\right)$. Therefore, $\min \left\{\varphi(x-z): z \in \overline{C\left(K^{\prime} y\right)}\right\} \geq \min \left\{\varphi(x-z): z \in C\left(W^{\prime} y\right)\right\}$ and the rest of the proof can be completed as before. The statement involving the maximum is proved similarly.

Since the root system $\Phi^{\prime}$ is of type $B_{n}$, the argument in Example 4.3 applies to show that Cheng's algorithm is the same as ours. The other main results of Cheng in this case also follow from ours.

EXAMPLE 4.5. $\mathscr{M}$ is the set of symmetric $n \times n$ matrices over $\mathbb{C}$ and $\sim$ is unitary congruence: $X \sim Y$ if and only if $X=U Y U^{t}$ for some $U \in U(n)$ (cf. [C, type (IV), p. 171]).

Let $G=S p(n, \mathbb{R})=\left\{g \in S L(2 n, \mathbb{R}): g^{t} J_{n, n} g=J_{n, n}\right\}$, where $J_{n, n}=\left[\begin{array}{cc}0 & I_{n} \\ -I_{n} & 0\end{array}\right]$, and let $K=\left\{\left[\begin{array}{cc}a & b \\ -b & a\end{array}\right]: a, b \in \mathbb{R}_{n \times n}, a+i b \in U(n)\right\}$. We have $\mathfrak{g}=\left\{\left[\begin{array}{cc}a & b \\ c & -a^{\prime}\end{array}\right]\right.$ : $\left.a, b, c \in \mathbb{R}_{n \times n}, b^{t}=b, c^{t}=c\right\}, \mathfrak{k}=\left\{\left[\begin{array}{cc}a & b \\ -b & a\end{array}\right] \in \mathfrak{g}: a^{t}=-a, b^{t}=b\right\}$, and $\mathfrak{p}=\left\{\left[\begin{array}{cc}a & b \\ b & -a\end{array}\right] \in \mathfrak{g}: a^{t}=a, b^{t}=b\right\}$. The map $\left[\begin{array}{cc}a & b \\ -b & a\end{array}\right] \mapsto a+i b$ identifies $K$ with $U(n)$ and $\mathfrak{p}$ with the set of symmetric $n \times n$ matrices over $\mathbb{C}$, and with these identifications, congruence in $\mathfrak{p}$ under the adjoint action of $K$ is precisely unitary congruence. For $\mathfrak{a}$, we can take $\left\{\operatorname{diag}\left(x_{1}, \ldots, x_{n},-x_{1}, \ldots,-x_{n}\right)\right\}$, which identifies with $\mathbb{R}^{n}$ in the obvious way. The reduced root system $\Phi$ of the pair $(\mathfrak{g}, \mathfrak{a})$ is of type $C_{n}$. Therefore, an argument very similar to that given in Example 4.3 for the case of the root system $B_{n}$ shows that Cheng's algorithm for this example and ours coincide.

EXAMPLE 4.6. $\mathscr{M}$ is the set of $n \times n$ skew symmetric matrices over $\mathbb{C}$ and $\sim$ is unitary congruence (cf. [C, type (V), $\mathbb{F}=\mathbb{C}$, p. 171]).

Let $G=S O^{*}(2 n)=\left\{g \in S U(n, n): g^{t} L_{n, n} g=L_{n, n}\right\}$, where $L_{n, n}=\left[\begin{array}{cc}0 & I_{n} \\ I_{n} & 0\end{array}\right]$, and $K=\left\{\left[\begin{array}{ll}U & 0 \\ 0 & \bar{U}\end{array}\right]: U \in U(n)\right\}$. We have $\mathfrak{g}=\left\{\left[\begin{array}{cc}a & b \\ -\bar{b} & \bar{a}\end{array}\right]: \bar{a}^{t}=-a, b^{t}=-b\right\}, \mathfrak{k}$ consists of those matrices in $\mathfrak{g}$ with $b=0$, and $\mathfrak{p}$ those with $a=0$ (see [Kn, p. 367]). Identifying $K$ with $U(n)$ via $\left[\begin{array}{ll}U & 0 \\ 0 & \frac{U}{U}\end{array}\right] \mapsto U$, and $\mathfrak{p}$ with the set of $n \times n$ skew symmetric matrices via $\left[\begin{array}{cc}0 & b \\ -\bar{b} & 0\end{array}\right] \mapsto b$, we see that congruence in $\mathfrak{p}$ under the adjoint action of $K$ is precisely unitary congruence. For $\mathfrak{a}$, we may take all matrices $\left[\begin{array}{cc}0 & b \\ -\bar{b} & 0\end{array}\right]$ with $b$ of the 
form $\sum_{i=1}^{[n / 2]} x_{i}\left(e_{2 i-1,2 i}-e_{2 i, 2 i-1}\right)\left(x_{i} \in \mathbb{R}\right)$, which we identify with $\mathbb{R}^{[n / 2]}$ by sending the indicated matrix to $\left(x_{1}, \ldots, x_{[n / 2]}\right)$. Here we take $B(x, y)=\frac{1}{4} \operatorname{Re} \operatorname{Tr}(x y)$, so that restriction of $B$ to $a$ yields the standard inner product on $\mathbb{R}^{[n / 2]}$. Then the reduced root system $\Phi$ of the pair $(\mathfrak{g}, \mathfrak{a})$ is of type $C_{n / 2}$ if $n$ is even and of type $B_{(n-1) / 2}$ if $n$ is odd. Therefore, the comparison of Cheng's algorithm with ours given in Example 4.3 applies here as well.

EXAMPLE 4.7. $\mathscr{M}$ is the set of $n \times n$ skew symmetric matrices over $\mathbb{R}$ and $\sim$ is orthogonal similarity (cf. [C, type(V), $\mathbb{F}=\mathbb{R}$, p. 171]).

For this last example, we will use the version of Theorem 3.12 given in Corollary 3.14 (with a minor adjustment). Let $U=S O(n)^{\circ}$. Then $\mathfrak{u}=\mathfrak{s o}(n)$, which is the set of $n \times n$ skew symmetric matrices over $\mathbb{R}$. Note that congruence in $u$ under the adjoint action of $U$ is orthogonal similarity. We may take $\mathfrak{t}=\left\{\sum_{i=1}^{n / 2]} x_{i}\left(e_{2 i-1,2 i}-e_{2 i, 2 i-1}\right)\right.$ : $\left.x_{i} \in \mathbb{R}\right\}$ identified with $\mathbb{R}^{[n / 2]}$ by sending the indicated matrix to $\left(x_{1}, \ldots, x_{[n / 2]}\right)$. If $n$ is odd, then the reduced root system $\Phi$ of the pair $(\mathfrak{u}, \mathfrak{t})$ is $\Phi=\left\{ \pm e_{i} \pm e_{j}: i \neq\right.$ $j\} \cup\left\{ \pm e_{i}\right\}$ (signs read independently here and below), which is of type $B_{n}$, and taking $\Delta=\left\{e_{i}-e_{i+1}(1 \leq i<n), e_{n}\right\}$ we have $\mathfrak{t}^{+}=\left\{x \in \mathfrak{t}: x_{1} \geq \cdots \geq x_{n} \geq 0\right\}$. If $n$ is even, then $\Phi=\left\{ \pm e_{i} \pm e_{j}: i \neq j\right\}$, which is of type $D_{n}$, and taking $\Delta=\left\{e_{i}-e_{i+1}(1 \leq i<n), e_{n-1}+e_{n}\right\}$, we have $\mathfrak{t}^{+}=\left\{x \in \mathfrak{t}: x_{1} \geq \cdots \geq x_{n-1} \geq\left|x_{n}\right|\right\}$.

Let $U^{\prime}=O(n)$. Then $U^{\prime}=U D$, where $D$ is the set of diagonal matrices with diagonal entries \pm 1 . Now $D$ stabilizes $\mathfrak{t}$, so it makes sense to form the group $W^{\prime}$ generated by $W$ and $D$. $W^{\prime}$ is a finite reflection group associated with the root system $\Phi^{\prime}=\left\{ \pm e_{i} \pm e_{j}: i \neq j\right\} \cup\left\{ \pm e_{i}\right\}$ which is of type $B_{n}$. The set $\Delta^{\prime}=\left\{e_{i}-e_{i+1}(1 \leq\right.$ $\left.i<n), e_{n}\right\}$ is a base for $\Phi^{\prime}$ and the corresponding fundamental domain for the action of $W^{\prime}$ on $\mathfrak{t}$ is $\mathfrak{t}^{+^{\prime}}=\left\{x \in \mathfrak{t}: x_{1} \geq \cdots \geq x_{n} \geq 0\right\}$. Given $x \in \mathfrak{u}$, denote by $\bar{x}^{\prime}$ the unique element of $\mathfrak{t}^{+\prime} \cap U^{\prime} x$. Arguing as in Example 4.4, we see that the version of Theorem 3.12 given in Corollary 3.14 is valid with all notation replaced by the corresponding primed notation. Therefore, Cheng's results follow from ours just as in the earlier examples.

REMARK. Our reason for considering the group $K^{\prime}$ in Example 4.4 and the group $U^{\prime}$ in Example 4.7 was that we were interested in recovering Cheng's results. Of course, we get new results by considering the actions of just $K$ and $U$. For instance, in Example 4.4 we see that if $\mathscr{M}$ is the set $\mathbb{R}_{m \times n}$ of $m \times n$ matrices over $\mathbb{R}$ and $\sim$ is special orthogonal equivalence ( $X \sim Y$ if and only if $X=U Y V$ for some $U \in S O(m), V \in S O(n))$, then the results of Cheng apply unchanged to this setting if $m \neq n$ (for then $\Phi$ is of type $B_{l}$, where $l=\min \{m, n\}$ ), while new formulas are required if $m=n$ (for then $\Phi$ is of type $D_{n}$ ). In the latter case, the longest element $w_{0}$ of the Weyl group $W$ acts on a by the rule $w_{0} x=\left(-x_{1}, \ldots,-x_{n-1},(-1)^{n+1} x_{n}\right)$, so the statement about the maximum in Cheng's Theorem $2(a)(\mathbb{F}=\mathbb{R})$ changes slightly 
in this setting if $n$ is odd. Also, if $m=n$, then Cheng's algorithm in Theorem 1 must be changed to handle this setting. It is not too difficult to see that by making appropriate choices for the sets $I_{k}$ in Algorithm 2.4, one can produce an algorithm that bears some resemblance to Cheng's, but we feel that to include such an algorithm here would serve no real purpose since, for instance, the algorithm arising from the natural choices given after Algorithm 2.4 would be easier to implement on a computer (and, once implemented, would require only a change of Cartan matrix to be applicable to settings with other root systems).

\section{References}

[AB] M. F. Atiyah and R. Bott, 'The Yang-Mills equations over Riemann surfaces', Philos. Trans. Roy. Soc. Lond. Ser. A 308 (1982), 523-615.

[BGr] C. T. Benson and L. C. Grove, Finite reflection groups (Springer, New York, 1985).

[BGe] F. A. Berezin and I. M. Gel'fand, 'Some remarks on the theory of spherical functions on symmetric Riemannian manifolds', Tr. Mosk. Mat. Obshch. 5 (1956), 311-351 (in Russian). English transl. in Amer. Math. Soc. Transl. 221 (1962), 193-238.

[C] C. M. Cheng, 'An approximation problem with respect to symmetric gauge functions with application to matrix spaces', Linear and Multilinear Algebra 29 (1991), 169-180.

[H1] J. E. Humphreys, Introduction to Lie algebras and representation theory (Springer, New York, 1972).

[H2] Reflection groups and Coxeter groups (Cambridge University Press, Cambridge, 1990).

[Kn] A. Knapp, Lie groups beyond an introduction, Progress in Math. 140 (Birkhäuser, Boston, 1996).

[Ko] B. Kostant, 'On convexity, the Weyl group and Iwasawa decomposition', Ann. Sci. Ecole Norm. Sup. 6 (1973), 413-460.

[L] A. S. Lewis, 'Von Neumann's lemma and a Chevalley-type theorem for convex functions on Cartan subspaces', Research report (University of Waterloo, 1995).

[LT1] C. K. Li and N. K. Tsing, 'Norms that are invariant under unitary similarities and the $C$-numerical radii', Linear and Multilinear Algebra 24 (1989), 209-222.

[LT2] - 'Distance to the convex hull of the unitary orbit with respect to unitary similarity invariant norms', Linear and Multilinear Algebra 25 (1989), 93-103.

[MO] A. W. Marshall and I. Olkin, Inequalities: Theory of majorization and its applications (Academic Press, New York, 1979).

[R] M. Raîs, 'Remarks on a theorem of R. Westwick', Technical Report.

[T] T. Y. Tam, 'A unified extension of two results of Ky Fan on the sum of matrices', Proc. Amer. Math. Soc. 126 (1998), 2607-2614.

[vN] J. von Neumann, 'Some matrix-inequalities and metrization of matrix-space', in Collected Works, Vol. 4 (Pergamon, New York, 1962), Tomsk. Univ. Rev. 1 (1937), 286-300.

Department of Mathematics

Auburn University

Auburn University AL 36849-5310

USA

e-mail: holmerr@auburn.edu, tamtiny@auburn.edu 